\title{
How the complex interplay between different blocks determines the isothermal crystallization kinetics of triple crystalline PEO-b-PCL-b-PLLA triblock terpolymers
}

Jordana K. Palacios ${ }^{a}$, Junpeng Zhao ${ }^{b, c}$, Nikos Hadjichristidis ${ }^{c *}$, Alejandro J. Müller ${ }^{a, d *}$

aPOLYMAT and Polymer Science and Technology Department, Faculty of Chemistry, University of the Basque Country UPV/EHU, Paseo Manuel de Lardizabal 3, 20018 Donostia-San Sebastián, Spain.

${ }^{b}$ Faculty of Materials Science and Engineering, South China University of Technology, Guangzhou 510640, People's Republic of China.

${ }^{\mathrm{c}}$ King Abdullah University of Science and Technology (KAUST), Physical Sciences and Engineering Division, KAUST Catalysis Center, Thuwal, Saudi Arabia.

dIKERBASQUE, Basque Foundation for Science, Bilbao, Spain.

* Corresponding authors

E-mails: alejandrojesus.muller@ehu.es, nikolaos.hadjichristidis@kaust.edu.sa 


\section{ABSTRACT}

PEO- $b$-PCL- $b$-PLLA triblock terpolymers are fascinating triple crystalline materials. In this work, the isothermal crystallization kinetics of these terpolymers evaluated by differential scanning calorimetry (DSC) is presented for the first time and compared to analogous PCL- $b$-PLLA diblock copolymers and to PLLA, PCL and PEO homopolymers. The results are complemented by in-situ SAXS/WAXS synchrotron experiments. One, two and three step crystallization protocols were employed to study the crystallization kinetics of the blocks. At PLLA block crystallization temperatures, both PCL and PEO molten chains caused a strong plasticizing effect on the PLLA block crystallization, and the overall crystallization rate of the PLLA block in the terpolymers was higher than that in the PLLA$b$-PCL diblock copolymers. In the case of the PCL block, the crystallization was followed after PLLA was fully crystallized (two step crystallization). A nucleating effect induced by the previously formed PLLA crystals was observed. However, an anti-plasticizing effect on PCL crystallization was detected if the sample is quenched directly from the melt to the PCL crystallization temperature (one step crystallization). Finally, the crystallization of the PEO block was followed after PLLA and PCL had fully crystallized (three step crystallization). The PEO crystallization rate highly decreased due to the confinement imposed by the previously formed PLLA and PCL crystals. Complex competitive effects such as plasticization, nucleation, anti-plasticization and confinement occurred during the isothermal crystallization of tricrystalline PEO- $b$-PCL- $b$-PLLA triblock terpolymers. 


\section{INTRODUCTION}

Block copolymer crystallization is of major interest in the field of polymer physics, and the number of publications and reviews published has increasingly grown in the past two decades. ${ }^{1-12}$ Recent advances in novel block copolymerization routes have allowed the production of different molecular architectures, such as linear and cyclic diblock and triblock copolymers and terpolymers, as well as more complicated chain topologies like stars and combs. ${ }^{13-14}$

Diblock and triblock copolymers and terpolymers with one or two crystallizable blocks have been investigated extensively. ${ }^{2-7,} 9-10,15-23$ It is well known that the crystallization of these materials is influenced by block composition, molecular weight and crystallization conditions, as well as segregation strength. However, the addition of a third crystallizable block raises the complexity of the crystallization behavior of these materials. Very recently, a limited number of publications have reported unique $\mathrm{ABC}$-type triblock terpolymers, in which the three blocks are able to crystallize upon cooling from the melt. ${ }^{24-26}$ In a previous publication, we reported the sequential crystallization and morphology of triple crystalline

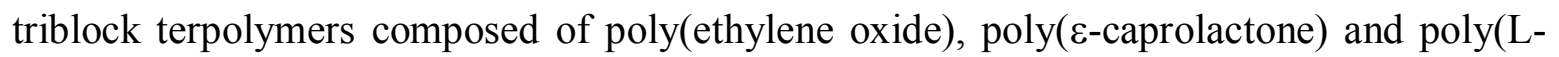
lactide) (PEO- $b$-PCL- $b$-PLLA) ${ }^{24}$ In this work, we pursue a deeper understanding of their crystallization behavior by performing isothermal crystallization experiments in these unique PEO-b-PCL- $b$-PLLA triblock terpolymers.

Block copolymers composed of biodegradable and biocompatible polymers, such as PLLA, PCL and PEO, have interesting potential applications in the biomedical field. ${ }^{2,27-28}$ Understanding their crystallization behavior is relevant to tune biodegradation, as well as their physical and mechanical properties. In the last decades, many authors have extensively 
studied and reviewed the isothermal crystallization behavior of PCL- $b$-PLLA diblock copolymers. ${ }^{29-35}$ In previous investigations, some of us ${ }^{31-32,36-38}$ have demonstrated a diluent effect caused by the PCL molten chains on the crystallization of the PLLA block. As the PCL content in the diblock copolymer increases, the PLLA crystallization and melting temperatures as well as crystallization rate decreased, in comparison to the PLLA homopolymer. Although, the depression of the crystallization rate was only significant when the PLLA content was lower than $10 \%$. In contrast, a more pronounced effect was observed in the crystallization rate of the PCL block after crystallizing the PLLA in two sequential steps. As the PLLA content increased, the supercooling greatly augmented and the PCL crystallization rate sharply decreased. The previously formed PLLA crystals forced the PCL chains to crystallize inside their limited interllamellar regions, restricting the crystallization process. The PCL block fractioned crystallization observed and the Avrami index, estimated close to 1 , account for a homogeneous nucleation process for this block. These are evidences of the confinement imposed by the covalently linked PLLA crystals on the PCL block crystallization. Despite that the PLLA crystals had a nucleation effect on PCL crystallization (proved by self-nucleation experiments), the crystallization kinetics was not enhanced because of the confinement effects.

The isothermal crystallization behavior of PEO- $b$-PLLA diblock copolymers is similar to that of the aforementioned PCL- $b$-PLLA ones. Several publications and reviews regarding the crystallization of this system account for that. ${ }^{6,27,39-44}$ As in the PCL- $b$-PLLA diblock copolymers, the miscible and molten PEO chains caused a plasticizing effect during PLLA isothermal crystallization. Regarding the PLLA crystallization rate, opposite effects have been observed. Some authors claimed that molten PEO chains improve the 
crystallizability of the PLLA block, ${ }^{40,45}$ while others reported a retarded PLLA crystallization due to the increased mobility of the bonded PEO molten chains. ${ }^{6,46}$ On the other hand, the crystallization kinetics of the PEO block exhibited a dependency with block composition. If the PLLA block length decreases, an increase in the PEO crystallization rate was observed. That increment may be due to a nucleating effect provided by the PLLA crystals. However, the previously formed PLLA crystals confined the crystallization of the PEO block at larger PLLA block lengths. ${ }^{40}$ Other authors ${ }^{47-48}$ also evaluated how the crystallization conditions of the PLLA block affect the subsequent crystallization of a PEO block. From their observations, higher PLLA crystallization temperatures and lower PLLA crystallization cooling rates enhanced the crystallization of the PEO block, as a result of an enlargement of the PEO microdomains.

Despite the extensive literature published regarding the crystallization behavior of double crystalline diblock copolymers, very few studies have successfully reported a fully triple crystalline $\mathrm{ABC}$-type triblock terpolymer. At room temperature, Sun et al. ${ }^{25}$ detected the triple crystalline nature of triblock and pentablock terpolymers composed of PLLA, PCL and PEO employing DSC and WAXS analysis. Recently, Chiang et al. ${ }^{26}$ reported single crystals of PEO- $b$-PCL- $b$-PLLA triblock terpolymers obtained from solution in thin films. Other authors have evaluated the ability of these amphiphilic terpolymers to form micellar structures ${ }^{49}$ or their potential use in drugs release applications. ${ }^{25}$ In a previous publication, some of us $^{24}$ reported triple crystalline PEO- $b$-PCL- $b$-PLLA triblock terpolymers with identical PEO and PCL block lengths and two different PLLA block lengths. ${ }^{50}$ DSC and WAXS measurements at very low cooling rates demonstrated that the three blocks are capable of crystallization in a specific sequence upon cooling from the melt. Due to the 
terpolymer composition, the PLLA block crystallized first, then the PCL block and finally the PEO block. As in the analogous diblock copolymers, the melt miscibility of the terpolymers was proven by SAXS measurements. Therefore, the phase separation is driven by PLLA crystallization that templates the spherulitic morphology of the terpolymer. The subsequent crystallization of the PEO and PCL blocks does not change the macroscopic superstructure and takes place inside the interlamellar regions of the previously formed PLLA crystals. Only a change in the magnitude of the birefringence is detected by Polarized Light Optical Microscopy (PLOM). ${ }^{24}$ A more detailed observation by AFM demonstrated the alternating lamellar structure of these terpolymers.

To our knowledge, this is the first time that the isothermal crystallization behavior of triple crystalline PEO-b-PCL- $b$-PLLA triblock terpolymers has been studied. In this work, a detailed study of the isothermal crystallization kinetics of the three PLLA, PCL and PEO blocks employing different crystallization protocols in a DSC is presented. Moreover, complementary in-situ SAXS/WAXS measurements were performed. The addition of a third crystallizable block certainly increases the complexity of the crystallization behavior as compared to double crystalline diblock copolymers. Complex competitive effects between the blocks, such as plasticizing, nucleation, anti-plasticizing and confinement will be discussed. Designing and tuning the properties of $\mathrm{ABC}$ terpolymers requires a deep comprehension of the crystallizability of each block, and of the influence of each crystalline phase over the other two.

EXPERIMENTAL 


\section{Materials}

Two triblock terpolymers and the analogous diblock copolymers and homopolymers were the samples employed to evaluate the crystallization behavior. The PEO- $b$-PCL- $b$-PLLA triblock terpolymers were synthesized by a one-pot sequential organocatalytic ring-opening polymerization of ethylene oxide (EO), e-caprolactone (CL) and L-lactide (LLA) using benzyl alcohol as the initiator and a phosphazene base, 1-tert-butyl-2,2,4,4,4pentakis(dimethylamino)-2 $2 \lambda^{5}, 4 \lambda^{5}$-catenadi(phosphazene) $\left(t-\mathrm{BuP}_{2}\right)$, as a single catalyst for the three monomers. More details regarding the synthesis can be found in reference. ${ }^{50}$ The triblock terpolymers have the same lengths of PEO and PCL blocks $\left(4600 \mathrm{~g} \mathrm{~mol}^{-1}\right.$ for PEO and $6800 \mathrm{~g} \mathrm{~mol}^{-1}$ for PCL), and different lengths of PLLA blocks (4700 and $8500 \mathrm{~g} \mathrm{~mol}^{-1}$, respectively). The corresponding PCL- $b$-PLLA diblock copolymers and PEO, PCL and PLLA homopolymers were synthesized similarly with the length of each component controlled by the feed ratio of monomer to initiator..$^{50-51}$

Size exclusion chromatography (SEC) revealed that the homopolymers, diblock copolymers and triblock terpolymers had relatively low molecular weight distributions (ĐM $<1.20)$, and the nuclear magnetic resonance spectra $\left({ }^{1} \mathrm{H}\right.$ NMR) represented all the characteristic signals of the expected macromolecular structure, including the main bodies of the blocks, end groups and groups linking different blocks. ${ }^{50}$ Due to the use of PEO standards, the number-average molecular weight $\left(M_{\mathrm{n}}\right)$ of the PEO obtained by SEC analysis was considered the absolute value, which was then used to calculate the $M_{n s}$ of the other blocks in the triblock copolymers from the ${ }^{1} \mathrm{H}$ NMR spectra (values given above). $M_{\mathrm{n}}$ of PCL, PLLA homopolymers and block lengths of the PCL- $b$-PLLA diblock copolymers were calculated 
from ${ }^{1} \mathrm{H}$ NMR spectrum by comparison of the signal integrals of the benzyl end group and monomeric units.

All the samples are described in Table 1. The subscript numbers represent the weight fractions of the blocks calculated from the $M_{n S}$ and the superscript numbers, the molecular weight of the entire sample.

Table 1. Block molecular weight $\left(M_{n}\right)$ and polidispersity index (PDI) of the terpolymers, diblock copolymers and homopolymers.

\begin{tabular}{lllll}
\hline Sample code & $\begin{array}{l}M_{n} \text { PEO block } \\
\left(\mathrm{g} \mathrm{mol}^{-1}\right)\end{array}$ & $\begin{array}{l}M_{n} \text { PCL block } \\
\left(\mathrm{g} \mathrm{mol}^{-1}\right)\end{array}$ & $\begin{array}{l}M_{n} \text { PLLA block } \\
\left(\mathrm{g} \mathrm{mol}^{-1}\right)\end{array}$ & $\begin{array}{l}\text { PDI } \\
(\mathrm{Mw} / \mathrm{Mn})\end{array}$ \\
\hline PEO $^{3.8}$ & 3800 & - & - & 1.03 \\
PCL $^{7}$ & - & 7000 & - & 1.10 \\
PLLA $^{4.6}$ & - & - & 4600 & 1.10 \\
PLLA $^{8.6}$ & - & - & 8600 & 1.12 \\
PCL $_{59}$ PLLA $_{41}{ }^{11.2}$ & - & 6600 & 4600 & 1.21 \\
PEO $_{29}$ PCL $_{42}$ PLLA $_{29}{ }^{16.1}$ & 4600 & 6800 & 4700 & 1.10 \\
PCL $_{43}$ PLLA $_{57}{ }^{15.4}$ & - & 6600 & 8800 & 1.16 \\
PEO $_{23}$ PCL $_{34}$ PLLA $_{43}{ }^{19.9}$ & 4600 & 6800 & 8500 & 1.18 \\
\hline
\end{tabular}

\section{Isothermal crystallization followed by differential scanning calorimetry (DSC)}

A Perkin Elmer DSC Pyris 1 was employed to perform DSC measurements of the homopolymers, diblock copolymers and triblock terpolymers. Samples of approximately 3 mg were encapsulated in aluminum pans and tested under ultra-high purity nitrogen atmosphere. The instrument was previously calibrated with an indium standard. The isothermal crystallization of each block in the diblock copolymers and terpolymers was studied separately. In all cases, preliminary tests were performed to ensure that the block under study did not crystallize during the scans previous to the isothermal step. Several 
crystallization temperatures were employed and the crystallization process of each block as function of time was recorded..$^{52}$

\section{The PLLA block}

The sample was first melted at $160^{\circ} \mathrm{C}$ during $3 \mathrm{~min}$ and next cooled down until $0{ }^{\circ} \mathrm{C}$ at 20 ${ }^{\circ} \mathrm{C} \min ^{-1}$ (to improve the nucleation density of the PLLA block), and kept at that temperature

for $1 \mathrm{~min} .{ }^{53}$ Then, it was heated up to the PLLA crystallization temperature $\left(T_{c}\right)$ at $60^{\circ} \mathrm{C} \mathrm{min}{ }^{-}$ 1. It should be noted that both PCL and PEO blocks are molten in the $T_{c}$ range of the PLLA block. The sample was isothermally crystallized until saturation and finally it was heated again from $T_{c}$ to $160{ }^{\circ} \mathrm{C}$ at $20^{\circ} \mathrm{C} \mathrm{min}^{-1}$.

\section{The PCL block}

To study the crystallization kinetics of the PCL block, two different approaches were employed: two step and one step crystallization. In the first one, the PLLA block was first crystallized until saturation, as it was described previously. At those temperatures, the PCL and the PEO blocks are molten. After PLLA crystallization was completed, the sample was rapidly cooled at $60{ }^{\circ} \mathrm{C} \mathrm{min}^{-1}$ until the PCL crystallization temperature. Then, the PCL block was isothermally crystallized until saturation and finally, the sample was heated again at 20 ${ }^{\circ} \mathrm{C} \min ^{-1}$.

In the second approach, the crystallization process was carried out in one step. The sample was first melted at $160^{\circ} \mathrm{C}$ during 3 min. After that, it was cooled down at $60{ }^{\circ} \mathrm{C}$ min $^{-}$ 
${ }^{1}$ until the PCL crystallization temperature and isothermally crystallized until saturation. Finally, the subsequent heating scan a $20^{\circ} \mathrm{C} \mathrm{min}^{-1}$ was recorded.

\section{The PEO block}

The isothermal crystallization of the PEO block in the terpolymers was followed employing a three step thermal protocol. After melting the sample during $3 \mathrm{~min}$ at $160^{\circ} \mathrm{C}$, the PLLA block was crystallized until saturation as previously described. Then, the sample was rapidly cooled down, and the PCL block was fully crystallized at a temperature high enough to keep the PEO block molten. After PCL crystallization was completed, the sample was quenched again until PEO crystallization temperature. The PEO block was allowed to crystallize until saturation. Finally, the subsequent heating scan at $20^{\circ} \mathrm{C} \mathrm{min}-1$ was recorded.

\section{Simultaneous wide angle and small angle X-Ray scattering (SAXS/WAXS)}

In-situ simultaneous SAXS/WAXS measurements were carried out at the beamline BL11NCD in the ALBA Synchrotron Radiation Facility (Cerdanyola del Valles, Barcelona, Spain). The samples were tested inside DSC pans and the scattering of an empty DSC pan

was subtracted from the data. The wavelength of the radiation source was $\lambda=1 \AA$. WAXS scattering profiles were recorded using a Rayonix LX255-HS detector with a resolution of 1920x5760 pixels (pixel size: $44 \mu^{2}$ ). The sample-to-detector distance was $132.6 \mathrm{~mm}$, the effective scattering vector $q$ range was $8-22 \mathrm{~nm}^{-1}$, and the tilt angle $21.2^{\circ}$. SAXS patterns were collected using an ADSC Q315r detector with a resolution of 3070x3070 pixels (pixel size: $102 \mu \mathrm{m}^{2}$ ). The sample-to-detector distance was $6495.0 \mathrm{~mm}$, covering a scattering vector 
$q$ range from 0.1 to $2.7 \mathrm{~nm}^{-1}$. The tilt angle was $0^{\circ}$. The calibration was performed employing silver behenate and $\mathrm{Cr}_{2} \mathrm{O}_{3}$ standards. The temperature profile was controlled by a Linkam Scientific Instruments THMS600 stage coupled to a liquid nitrogen cooling system. The acquisition time for each pattern was $6 \mathrm{~s}$. The two dimensional scattering patterns were integrated radially to one dimensional intensity profiles using the program pyFAI, as a function of the magnitude of the scattering vector, $q=2 \pi / \mathrm{d}=4 \pi \sin \theta / \lambda$.

\section{Morphological observations}

The morphology was observed by polarized light optical microscopy (PLOM) and atomic force microscopy (AFM). For PLOM, films were prepared by melting the sample between a glass slide and a cover slip employing a Mettler Toledo FP82HT hot stage plate. Samples were observed in an Olympus BX51 microscope, equipped with digital camera Olympus SC50, crossed polarizers and making use of $\lambda$ wave plate to determine the sign of the spherulites. The thermal protocol applied was as follows: once the sample was melted at $160^{\circ} \mathrm{C}$ inside the hot stage, it was kept at this temperature for $3 \mathrm{~min}$. Then, it was quickly cooled down to $100{ }^{\circ} \mathrm{C}$, and isothermally crystallized at this temperature. Finally, the sample was cooled down to room temperature.

For AFM observations, a Digital Instruments multimode scanning probe microscope equipped with a Nanoscope IV controller was employed. The figures were acquired in tapping mode using microfabricated silicon tips/cantilevers (cantilever spring constant, $\mathrm{k}=$ $42 \mathrm{~N} / \mathrm{m}$, and resonance frequency, $\mathrm{f}_{\mathrm{o}}=320 \mathrm{kHz}$, Bruker). Height and phase images of lamellae were collected simultaneously and were subjected to a first-order plane-fitting 
procedure to compensate for sample tilt. The samples were prepared by spin-coating into a homogeneous thin film on a mica substrate from chloroform solution $(50 \mathrm{mg} / \mathrm{mL})$.

\section{RESULTS AND DISCUSSION}

Producing a triple crystalline morphology in linear triblock terpolymers depends on the crystallization conditions, the segregation strength, the molecular weight, and the order of the blocks within the terpolymer, among others. In the case of melt miscible terpolymers, the block that crystallizes first upon cooling from the melt (at higher temperatures) will template the entire terpolymer superstructural morphology. The position of this block within the terpolymer (whether it is the end block or the middle block) will probably affect its crystallization kinetics. In this paper, we focus on the isothermal crystallization of PEO- $b$ PCL- $b$-PLLA triblock terpolymers and compare their behavior with their PCL- $b$-PLLA diblock precursors. Although, analogous PEO- $b$-PLLA- $b$-PCL terpolymers would also be highly interesting to study the effect of PLLA block chain tethering, they were not considered for this first work due to synthetic difficulties, however, they will be the subject of future work once they can be prepared.

The morphology and sequential crystallization of two PEO- $b$-PCL- $b$-PLLA triblock terpolymers of different composition were reported in a previous publication by some of us ${ }^{24}$ and it is summarized in Figure 1. Both terpolymers exhibited a homogeneous melt at $160{ }^{\circ} \mathrm{C}$ state as confirmed by SAXS measurements (see Figure 1a for $\mathrm{PEO}_{23} \mathrm{PCL}_{34} \mathrm{PLLA}_{43}{ }^{19.9}$ sample). A single glass transition temperature is also an evidence of miscibility. However, for all the diblock copolymers and terpolymers, it was difficult to properly measure the glass transition temperature in the subsequent heating scans after quenching the sample from the 
melt. The glass transition was closely followed by cold crystallization of the PCL block, and it seems to take place in a range between -60 and $-30{ }^{\circ} \mathrm{C}$ in all the samples (see Figure $\mathrm{S} 1$ in Supporting Information).

Upon cooling, the PLLA block in the terpolymers crystallizes first and drives the phase separation. WAXS measurements confirmed the crystallization order: first the PLLA block, then the PCL block, and finally the PEO block (see Figure 1b). The crystallization of each block separately was also observed by DSC experiments conducted at a low cooling rate (1 ${ }^{\circ} \mathrm{C} \mathrm{min}^{-1}$ ) (see Figure 1c). Since PEO and PCL have very similar crystallization and melting temperatures, it was possible to elucidate from WAXS analysis that the middle exothermic peak in Figure 1c corresponded to the PCL block (see green arrow in Figure 1c) while the lower temperature one to the PEO block (see blue arrow in Figure 1c). Due to the melt miscibility of these terpolymers, the morphology is templated by the first crystallization of the PLLA block. The subsequent crystallization of the PCL and PEO block did not modify significantly the superstructure, and only a sequential change in the magnitude of the birefringence took place after the crystallization of the other two blocks. The final morphology consisted in a mixed spherulitic-type superstructure composed of PLLA, PCL and PEO lamellae (see Figure 1d and e). ${ }^{24}$

The schemes presented in Figure 1f and 1g illustrate the superstructural morphology and the lamellar arrangement in the triblock terpolymers under study ${ }^{24}$. 

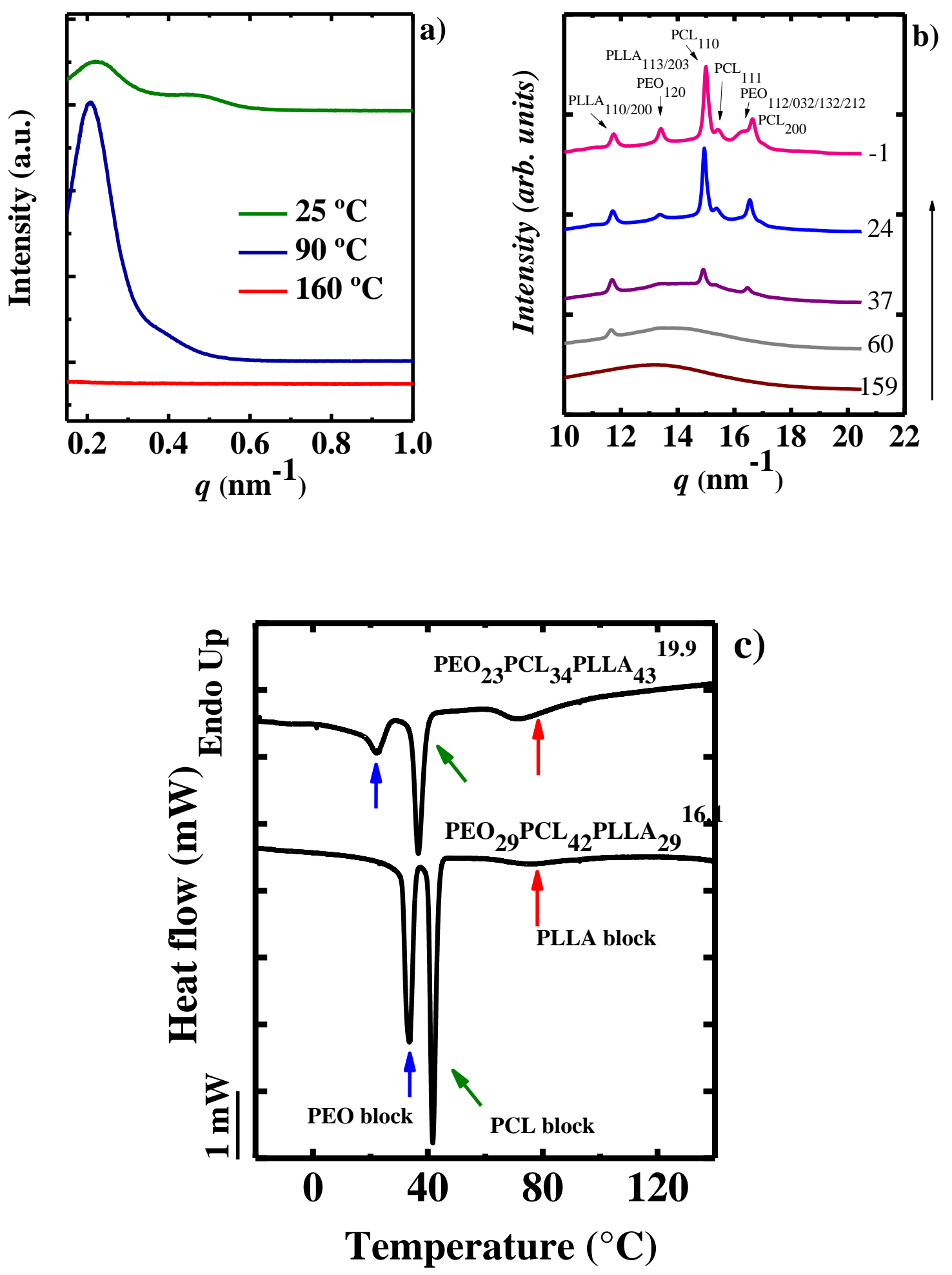

c) 
d)

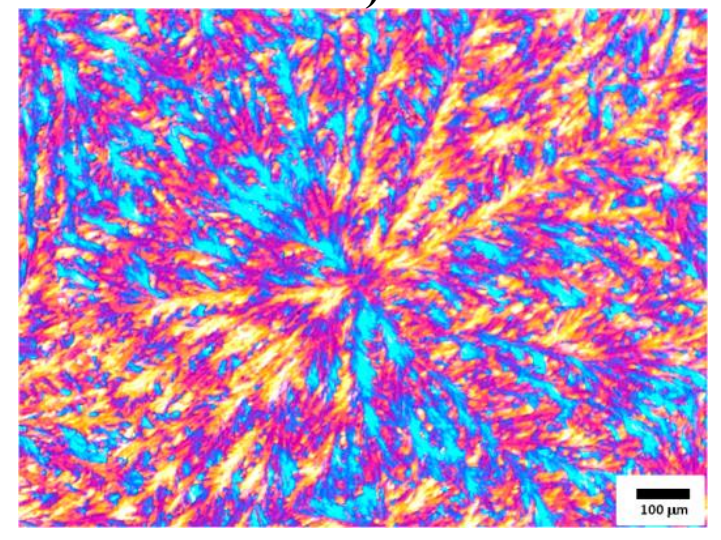

f)

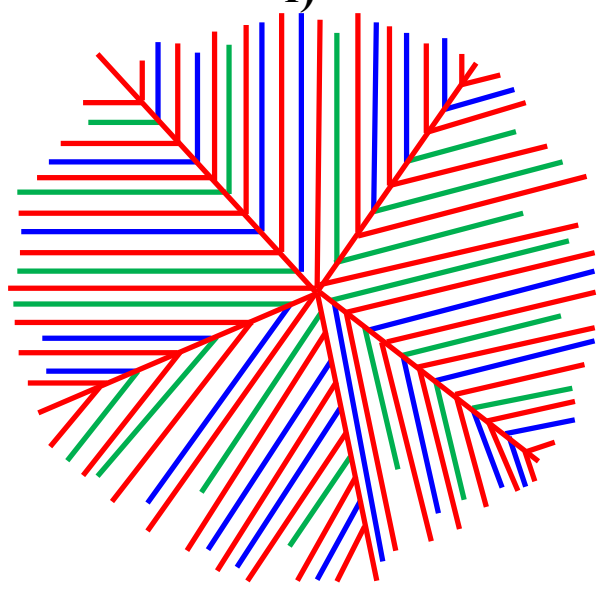

e)

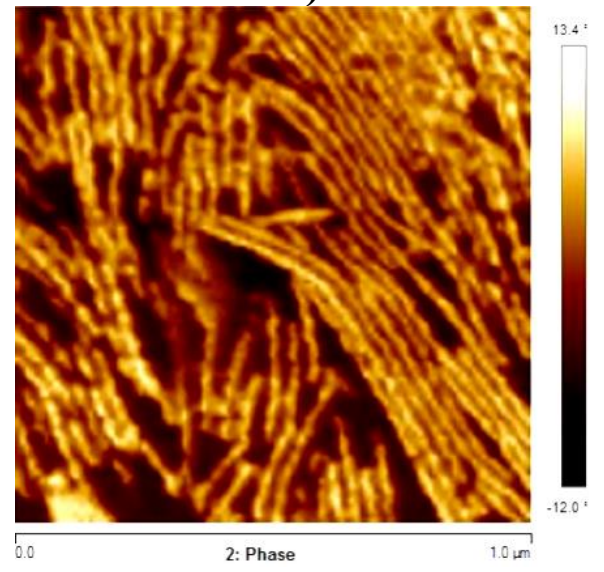

g)

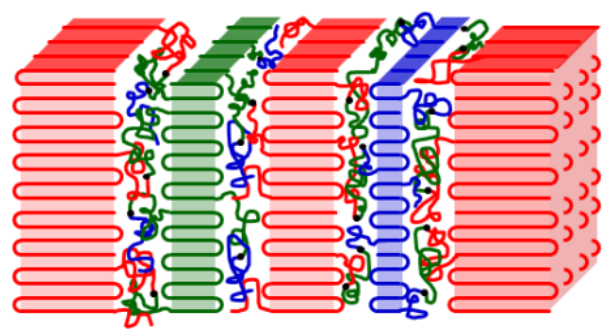

Figure 1. a) SAXS patterns taken at different temperatures on heating of $\mathrm{PEO}_{23} \mathrm{PCL}_{34} \mathrm{PLLA}_{43}{ }^{19.9} \mathrm{~b}$ )WAXS patterns taken at different temperatures during cooling from the melt at $5{ }^{\circ} \mathrm{C} \mathrm{min}{ }^{-1}$ of $\mathrm{PEO}_{29} \mathrm{PCL}_{42} \mathrm{PLLA}_{29}{ }^{16.1} \mathrm{c}$ ) DSC cooling scans at $1{ }^{\circ} \mathrm{C} \mathrm{min}{ }^{-1}$ after melting at $160{ }^{\circ} \mathrm{C}$ for $3 \mathrm{~min}$ d) PLOM micrographs taken at room temperature ofPEO ${ }_{29} \mathrm{PCL}_{42} \mathrm{PLLA}_{29}{ }^{16.1}$ after crystallizing first the PLLA block at $100^{\circ} \mathrm{C}$, e) AFM phase micrographs of $\mathrm{PEO}_{23} \mathrm{PCL}_{34} \mathrm{PLLA}_{43} 3^{19.9}$ observed at $25^{\circ} \mathrm{C}$. The sample was quenched to 25 ${ }^{\circ} \mathrm{C}$ after isothermal crystallization in three steps: first at $80^{\circ} \mathrm{C}$, then at $45^{\circ} \mathrm{C}$, and finally at 28 ${ }^{\circ} \mathrm{C}$, f) Schematic representation of the spherulitic morphology and $\mathrm{g}$ ) the trilayered morphology at the nanoscale in the triple crystalline PEO- $b$-PCL- $b$-PLLA triblock terpolymers. 
A color code was used in Figures 1f and 1g to represent each block: red for PLLA, green for PCL and blue for PEO. The peculiar disposition of the lamellae, where random insertion of PCL or PEO lamellae occurs in between PLLA lamellae has been demonstrated by SAXS, AFM and the simulation of the SAXS patterns in our previous work ${ }^{24}$.

In order to establish the influence of each block and the environment created in the crystallization behavior of the terpolymers, different crystallization protocols have been applied and the isothermal crystallization of each block has been followed.

\section{Overall crystallization kinetics of the PLLA block in the terpolymers and diblock copolymers}

It is well known that the crystallization kinetics of PLLA is slow ${ }^{54}$. In fact, it was not possible to follow the isothermal crystallization of the PLLA block in the terpolymers after cooling the sample from the melt to the crystallization temperature. The DSC isothermal signal was too small for detection. Therefore, in order to increase the nucleation density of PLLA, the sample was cooled from the melt until $0^{\circ} \mathrm{C}$ and kept at that temperature for $1 \mathrm{~min}$, and then, rapidly heated up to the PLLA crystallization temperature (see Figure 2). In this way, the isothermal cold crystallization of PLLA was followed. During the rapid cooling and heating steps, the PLLA block did not crystallize.

Thus, from the DSC isothermal experiments, the inverse of the half-crystallization time $\left(1 / \tau_{50 \%}\right)$ values were determined and plotted against the crystallization temperature. The $1 / \tau_{50 \%}$ values represent the inverse of the time needed to achieve the $50 \%$ of the total crystalline mass during the isothermal crystallization event, and represent an experimental measure of the overall crystallization rate. 


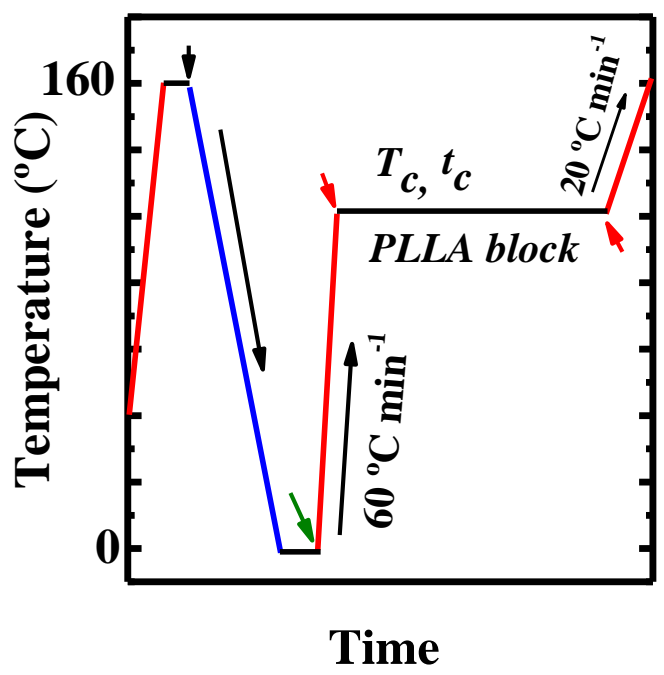

Figure 2. Thermal protocol applied to follow the isothermal crystallization behavior of the PLLA block.

Figure 3 compares the overall crystallization kinetics of the PLLA block in both terpolymers with that of the PLLA block in the analogous diblock copolymers. The length of the PLLA block is almost the same in the diblock copolymer and terpolymer. Following the isothermal cold crystallization of the corresponding PLLA homopolymers was not possible due to their very slow crystallization rate. The data in Figure 3 represents the left side of the typical bell-shape curve of the crystallization rate plot as a function of crystallization temperature $^{55}$. Thus, the crystallization rate of the samples decreased as the crystallization temperature reduces. The PLLA block crystallizes in a temperature range where both PCL and PEO blocks are in the melt. The molten PEO and PCL chains caused a strong plasticizing effect on the PLLA block crystallization. A higher supercooling was needed in order to crystallize the PLLA block at the same crystallization temperature (by extrapolation). The depression in $T_{c}$ was more notorious as the PLLA content in the terpolymer and the diblock 
copolymer was reduced (see Figure 3, left). In diblock copolymers of PLLA and PCL, and PLLA and PEO, a similar reduction in the PLLA crystallization temperature has been observed as the PLLA content (or molecular weight) decreases. ${ }^{36,40,45}$
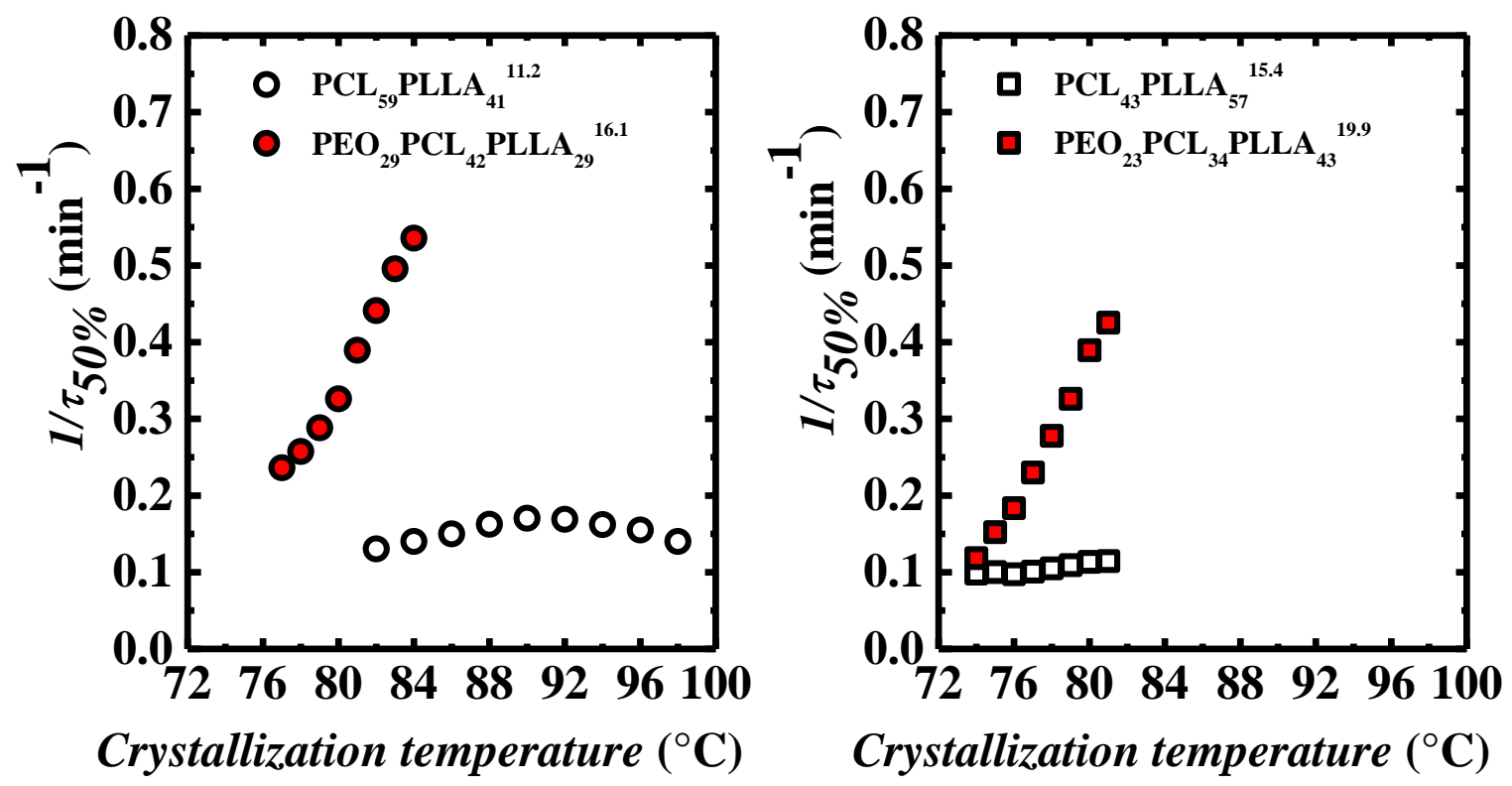

Figure 3. Inverse of half crystallization times $\left(1 / \tau_{50 \%}\right)$ versus crystallization temperature. Isothermal cold crystallization of the PLLA block within the diblock copolymers and terpolymers indicated.

Surprisingly, the overall crystallization rate of the PLLA block was higher in both terpolymers when compared to the crystallization of the PLLA block within the analogous diblock copolymers. This behavior was not expected, since in PCL- $b$-PLLA diblock copolymers, a decreased PLLA block crystallization rate has been reported ${ }^{36}$ However, the crystallization kinetics reported previously had been followed by cooling directly from the melt. ${ }^{36}$ In the present case, the cold isothermal crystallization has been determined upon heating from $0{ }^{\circ} \mathrm{C}$. Therefore, the left side of the bell-shape crystallization rate was examined. 
For the $\mathrm{PEO}_{29} \mathrm{PCL}_{42} \mathrm{PLLA}_{29}{ }^{16.1}$ triblock terpolymer (see Figure 3, left), the higher content of covalently bonded molten phase (PEO + PCL) compared to that in the diblock copolymer (only PCL) caused a strong plasticizing effect, shifting the crystallization rate curve to lower temperatures. Therefore, the increased crystallization rate might be related to the increase in the supercooling. The overall crystallization is the combination of the nucleation and growth events. At high supercoolings (left side of the bell-shape crystallization rate plot depicted in Figure 3), the crystallization kinetics is governed mainly by diffusion. The most plausible explanation of the enhanced overall crystallization rate is the presence of the additional highly flexible PEO block, as compared to the diblock copolymer. The high mobility of the mixed molten PEO block chains might aid the diffusion of the PLLA chains to the crystalline front, enhancing the PLLA crystallization kinetics in the terpolymers.

Additionally, the order of the blocks in the terpolymer is a factor of major importance that will probably affect the crystallization kinetics. In this case, the PLLA block is an end block in the terpolymer. If the PLLA block would have been in the middle, it is possible that the plasticizing effect over the PLLA crystallization could be even more pronounced.

The phase structure of the samples previous to the PLLA isothermal cold crystallization was examined by simultaneous SAXS/WAXS measurements. The PLLA block was crystallized upon heating from $0{ }^{\circ} \mathrm{C}$ up to the PLLA crystallization temperature. During the previous cooling scan down to $0{ }^{\circ} \mathrm{C}$, the PCL and PEO blocks can crystallize. Then, the PCL and PEO crystals melt in the subsequent heating scan up to the PLLA $T_{c}$. The PCL59PLLA $41^{11.2}$ diblock copolymer and $\mathrm{PEO}_{29} \mathrm{PCL}_{42} \mathrm{PLLA}_{29}{ }^{16.1}$ triblock terpolymer were selected to perform simultaneous SAXS/WAXS measurements during the isothermal crystallization step of the PLLA block. 

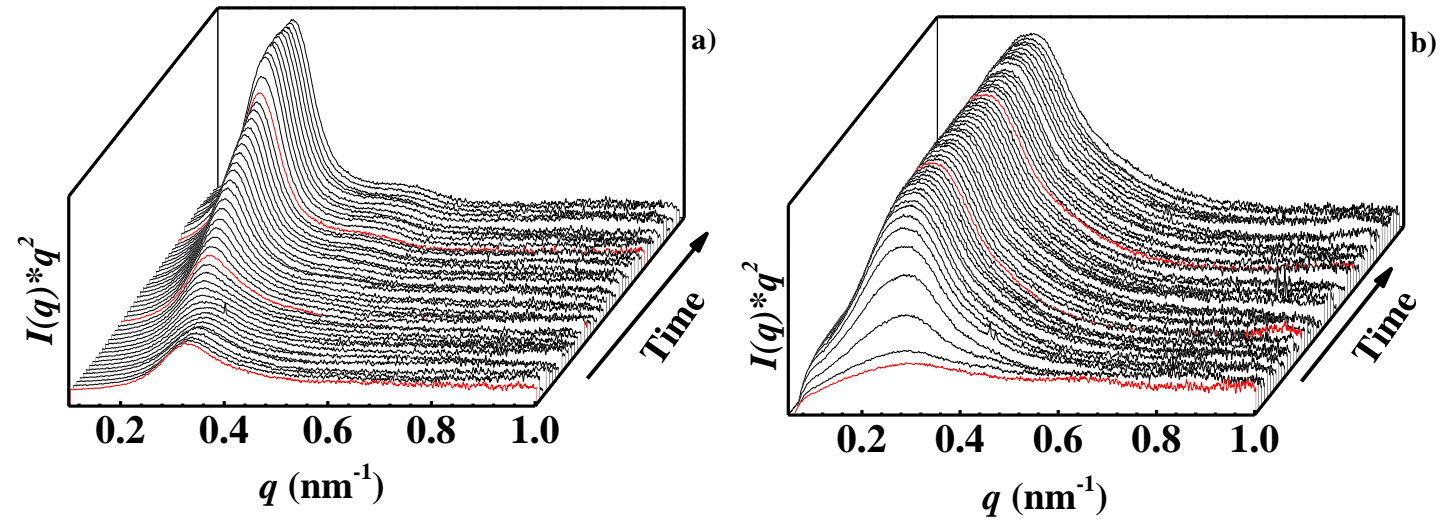

Figure 4. Lorentz corrected SAXS patterns during the isothermal cold crystallization of the PLLA block at $82{ }^{\circ} \mathrm{C}$ in a) PCL59PLA4 $41{ }^{11.2}$ diblock copolymer and b) $\mathrm{PEO}_{29} \mathrm{PCL}_{42} \mathrm{PLLA}_{29}{ }^{16.1}$ triblock terpolymer.

The same thermal protocol depicted in Figure 2 was applied and the isothermal cold crystallization of PLLA was carried out at $82{ }^{\circ} \mathrm{C}$ until saturation. The Lorentz corrected SAXS curves plotted against time are shown in Figure $4 \mathrm{a}$ for the PCL ${ }_{59} \mathrm{PLLA}_{41}{ }^{11.2}$ diblock copolymer and Figure $4 \mathrm{~b}$ for the $\mathrm{PEO}_{29} \mathrm{PCL}_{42} \mathrm{PLLA}_{29}{ }^{16.1}$ triblock terpolymer. The product between the intensity and the square of the scattering vector $(q)$ was plotted versus $q$.

At zero time, the PCL ${ }_{59} \mathrm{PLLA}_{41}{ }^{11.2}$ diblock copolymer exhibited a SAXS peak at $q=$ $0.33 \mathrm{~nm}^{-1}$ (see first red curve in Figure 4a). The scattering vector is related to the long period ( $d$-spacing) through the equation $d=2 \pi / q^{\max }$. This value corresponded to a long period value of $19.1 \mathrm{~nm}$. On the contrary, two broad and very diffuse scattering peaks were observed (at $q=0.32 \mathrm{~nm}^{-1}$ and $q=0.64 \mathrm{~nm}^{-1}$ ) in the $\mathrm{PEO}_{29} \mathrm{PCL}_{42} \mathrm{PLLA}_{29}{ }^{16.1}$ triblock terpolymer at the beginning of the isothermal crystallization step (see first red curve in Figure 4b). It seems that the crystallization of the PLLA block in the diblock copolymer started from a phase separated structure, while in the terpolymer, the PLLA block started to crystallize from a less 
segregated melt phase. To confirm that the samples were completely amorphous before the PLLA crystallization and to determine whether phase separation exists, SAXS/WAXS patterns were selected at specific times of the thermal protocol depicted in Figure 2 (see small arrows). The selected frames are shown in Figure 5.

At $160{ }^{\circ} \mathrm{C}$, both samples are molten. Thus, the WAXS patterns exhibited the characteristic amorphous halo and no melt structure was observed in the SAXS patterns measured at this temperature. The melt miscibility of PCL- $b$-PLLA diblock copolymers ${ }^{29,}$ $31-33,35,56$ and the specific PEO- $b$-PCL- $b$-PLLA terpolymers ${ }^{24}$ studied here has been previously reported. During the cooling until $0^{\circ} \mathrm{C}$, the PCL block crystallized in both samples (see WAXS profiles at $0{ }^{\circ} \mathrm{C}$ in Figure 5). A lamellar structure corresponding to the PCL crystalline lamellae was detected in the SAXS patterns (see the green plots on the right of Figure 5). The long period value of the lamellar arrangement was 19.7 and $17.4 \mathrm{~nm}$ for the diblock copolymer and terpolymer, respectively. Then, the PCL crystals melted during the subsequent heating scan up to $82^{\circ} \mathrm{C}$.

At the beginning of the PLLA crystallization (zero time) both samples were completely amorphous. No crystalline reflections were observed at this time in the WAXS plots of Figure 5 (left side, bottom red curves). However, the SAXS profile of the diblock copolymer showed a reflection prior to PLLA crystallization that was very diffuse in the SAXS profile of the terpolymer (see right side in Figure 5, bottom red curves). 

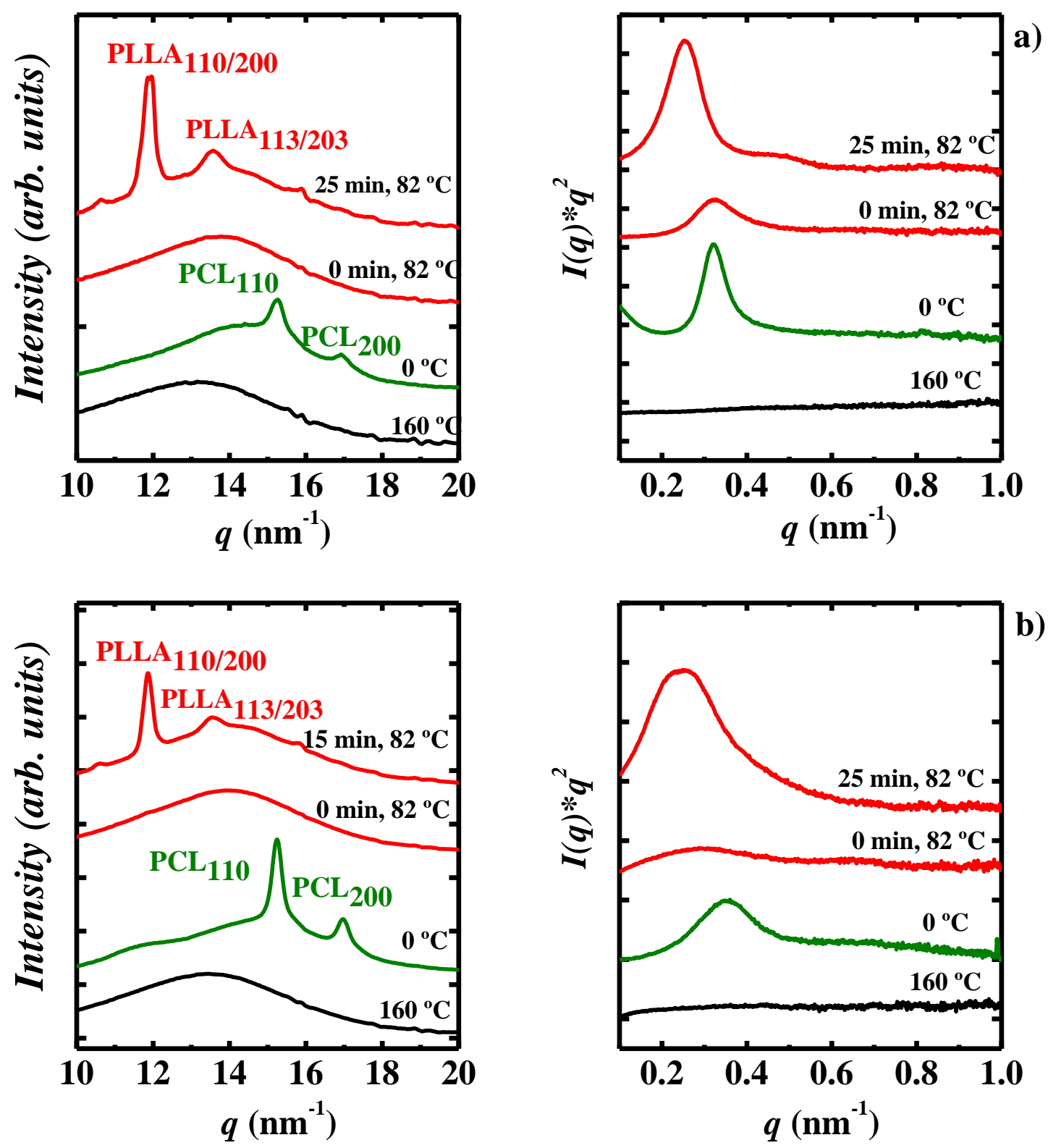

Figure 5. WAXS profiles (left) and Lorentz corrected SAXS profiles (right) of a) PCL59PLLA $4{ }_{11}^{11.2}$ diblock copolymer and b) $\mathrm{PEO}_{29} \mathrm{PCL}_{42} \mathrm{PLLA}_{29}{ }^{16.1}$ triblock terpolymer.

This reflection corresponded to a phase separation at $82{ }^{\circ} \mathrm{C}$, and described a lamellar structure with a long period of $19.1 \mathrm{~nm}$. This value is similar the one observed at $0{ }^{\circ} \mathrm{C}(19.7$ $\mathrm{nm}$ ) when the PCL block was crystallized. Thus, the SAXS reflection at $82{ }^{\circ} \mathrm{C}$ and 0 min might indicate a phase segregation of PCL amorphous lamellar microdomains that were 
crystalline before and a mixed PCL + PLLA amorphous phase. As a result, the PLLA crystallization initiated in the presence of a phase segregated structure. Due to the rapid heating, it was not possible to achieve a homogeneous phase after the melting of the PCL crystals and amorphous microdomains remained segregated. As for the $\mathrm{PEO}_{29} \mathrm{PCL}_{42} \mathrm{PLLA}_{29}{ }^{16.1}$ triblock terpolymer, the PLLA crystallization started from a less segregated melt phase. It is likely that the high mobility of the molten PEO block may have induced higher miscibility between the PCL and PEO chains and amorphous PLLA chains.

After the PLLA block was completely crystallized, the ordered lamellar phase prior to PLLA crystallization disappeared, as it was substituted by the PLLA crystalline lamellar structure. Similar observations have been reported by Hamley et al. ${ }^{31-32}$ in PLLA- $b$-PCL diblock copolymers but in their case the samples were crystallized upon cooling from melt. A transient ordered lamellar structure prior to PLLA crystallization was detected for $\mathrm{PLLA}_{60}{ }^{12} \mathrm{PCL}_{40}{ }^{9}$ and $\mathrm{PLLA}_{32}{ }^{7} \mathrm{PCL}_{68}{ }^{15}$, similar diblock copolymers to the one reported here. The size of the PLLA crystalline lamellar structure of both samples was similar. The values of the long period were 25.0 and $25.2 \mathrm{~nm}$ for the diblock copolymer and terpolymer, respectively. The reason is the similar PLLA block length in both samples (4600 and $4700 \mathrm{~g}$ $\mathrm{mol}^{-1}$ ). In the diblock copolymer, besides the first order reflection at $q=0.25 \mathrm{~nm}^{-1}$, a second order reflection was detected at $q=0.49 \mathrm{~nm}^{-1}$.

The length (or molecular weight) of the PLLA block in the terpolymers affects its crystallization rate. At the same temperature, as the PLLA block length increases the overall crystallization rate reduced slightly (see the terpolymers in Figure $3 a$ and $b$ ). It has been widely reported that the crystallization rate of PLLA tends to decrease as the molecular weight increases ${ }^{54,57}$ 
In order to compare the PLLA crystallinity achieved during the isothermal cold crystallization, the degree of crystallinity was calculated from the crystallization enthalpy obtained after crystallization saturates (see Figure 6).
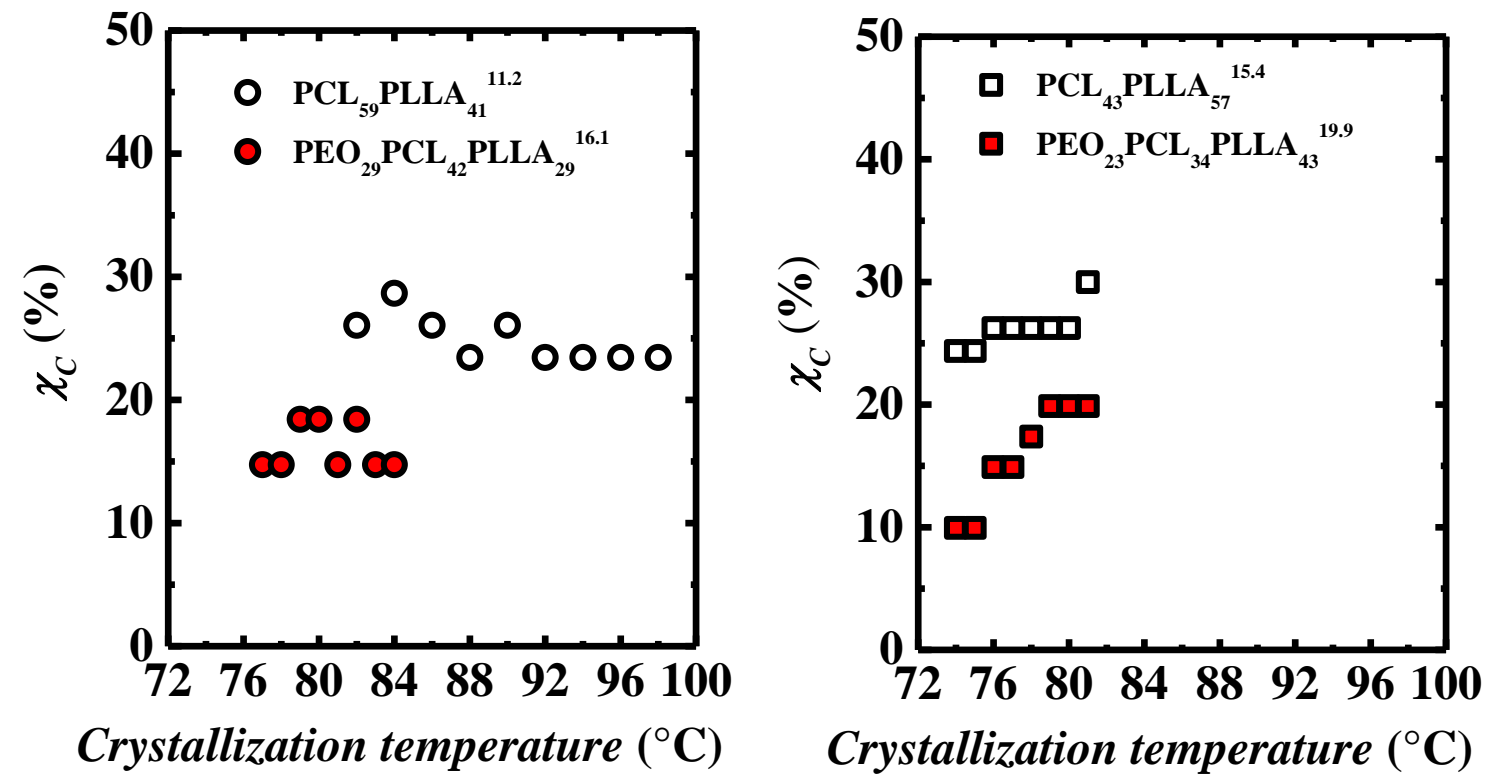

Figure 6. Crystallization degree $\left(\chi_{c}\right)$ of the isothermally crystallized PLLA block within the diblock copolymers and terpolymers indicated.

Both terpolymers have PLLA block crystallization degrees smaller than that of the corresponding PLLA block in the diblock copolymers. Even though the PLLA block in the terpolymer crystallizes faster, it does it to a lesser extend. The chain length of the PLLA block in the terpolymer is the same as in the diblock precursor. However, its relative composition among the blocks is lower. There is only 29 and $43 \%$ of PLLA in the terpolymers, in comparison to the 41 and $57 \%$ in the analogous diblock copolymers. Therefore, there is less PLLA phase available for crystallization in the sample as whole. In addition the content of plasticizing phase in the sample (i.e., PCL in the diblock copolymer 
and PCL + PEO in the terpolymer) will also affect the crystallizability of the PLLA block. In comparison with the quantity of PCL in the diblock copolymers, the content of the molten PEO + PCL phase in the terpolymers is higher $(71$ and $57 \%$ in the terpolymers versus 59 and $43 \%$ in the diblock copolymers). This fact caused a diluent effect that might have made more difficult the addition of new crystallizable segments to the crystallization front. The degree of crystallinity for the PLLA component in both terpolymers is between 10 and $20 \%$ while in the diblock copolymers it is between 20 and $30 \%$. The isothermal experimental data obtained from the DSC measurements was fitted to the Avrami equation following carefully the procedure proposed by Lorenzo at al. ${ }^{52}$. The crystallization kinetics in polymers have been described through several theories. ${ }^{55}$. One of them is the "free growth" theory formulated by Göler and Sachs ${ }^{58-61}$ that establishes once a given nuclei is initiated, it grows unrestrained or without the influence of others that may have also been nucleated or growing at the same time ${ }^{61}$. One of the possible solutions to this theory is the Avrami equation, also referred to as the Kolmogorov-Johnson-Mehl-Avrami equation ${ }^{62-66}$ :

$$
1-V_{c}\left(t-t_{0}\right)=\exp \left(-k\left(t-t_{0}\right)^{n}\right)
$$

This particular arrangement of the equation takes into account the role of the induction time, $t_{0}$, in the fitting. The other parameters are the relative volumetric transformed fraction $V_{c}$, the overall crystallization rate constant, $k$ (that includes both contributions of the crystallization process: nucleation and growth), and the Avrami index, $n$, which is to the combination of two terms, ${ }^{10}$ the time dependence of the nucleation $\left(n_{n}\right)$ and the crystal growth geometry $\left(n_{d}\right)$. In polymers with spherulitic type morphology (3D structure), the Avrami 
index expected is between 3 and 4; while in crystals growing with 2D aggregates, such as axialites, the Avrami values would be between 2 and 3. In both cases, the final value will depend on whether the nucleation event is sporadic or instantaneous. ${ }^{55,58,67}$ The quality of the fitting will depend on the conversion range $\left(V_{c}\right)$ employed since the Avrami equation rarely describes the complete process. In general, the equation fits the process properly until the primary crystallization ends, which is $<40-50 \% .{ }^{61}$

The Avrami index values, obtained from fitting the PLLA cold isothermal crystallization data, are plotted in Figure 7 against the crystallization temperature. The range of conversion employed in the fit was $3-20 \%$, and the correlation coefficients were always higher than 0.99 . The Avrami index of the diblock copolymers are around 2.5 , which is approximately 3 corresponding to instantaneously nucleated spherulites. The values of the terpolymers are smaller. The Avrami index of the terpolymer with the lower PLLA content $\left(\mathrm{PEO}_{29} \mathrm{PCL}_{42} \mathrm{PLLA}_{29}{ }^{16.1}\right)$ exhibited values close to 1.7 . This value agreed well with instantaneously nucleated axialites (or 2D aggregates). In addition, there appears to be a tendency of reducing the Avrami index with temperature in the PCL43 $\mathrm{PLLA}_{57}{ }^{15.4}$ diblock copolymer and $\mathrm{PEO}_{23} \mathrm{PCL}_{34} \mathrm{PLLA}_{43}{ }^{19.9}$ terpolymer. Increasing temperature should produce a more sporadic nucleation that should increase the Avrami index if growth dimensionality were constant. As in this case, the Avrami index decreases with temperature, such a decrease can only be due to the change in crystal dimension from $3 \mathrm{D}$ to $2 \mathrm{D}$, i.e., from spherulites to axialites, keeping the nucleation instantaneous. 

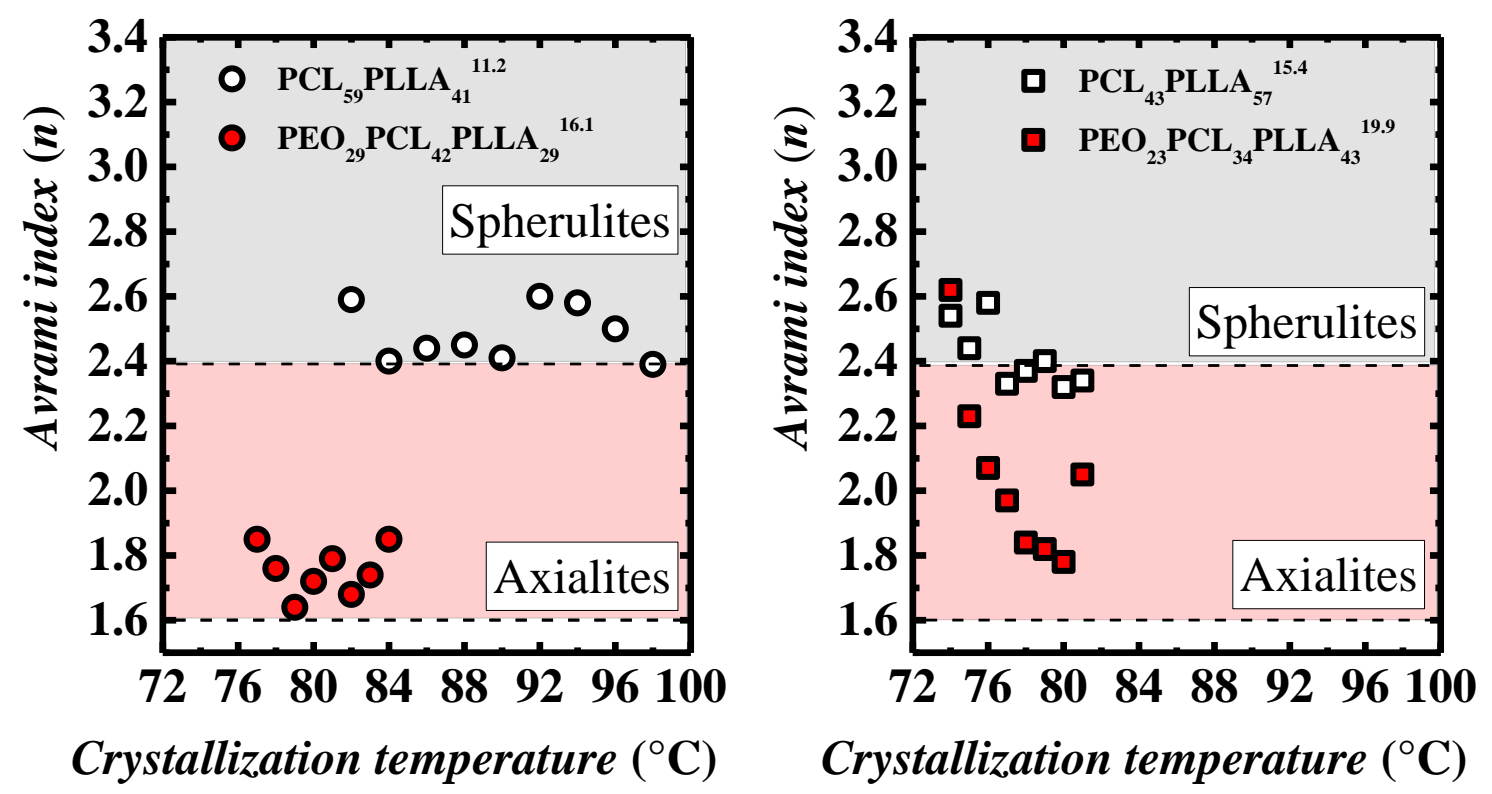

Figure 7. Avrami index ( $n$ ) values of the PLLA block within the diblock copolymers and terpolymers indicated.

Overall crystallization kinetics of the PCL block in the terpolymers and diblock copolymers

Since the PLLA block crystallizes first, two different crystallization protocols were employed to follow the crystallization behavior of the PCL block in the terpolymers and diblock copolymers. The results were compared to that of the PCL homopolymer of same molecular weight. In the first one (protocol 1), the PLLA block was first crystallized until saturation (after keeping the sample at $0{ }^{\circ} \mathrm{C}$ for $1 \mathrm{~min}$ ), and then, the sample was quenched until the crystallization temperature of the PCL block (see Figure 8). The PCL isothermal crystallization was registered. In the case of the terpolymers, the crystallization temperatures chosen were high enough to ensure that only the PCL block was crystallizing, while the PEO block remained molten. With this two step crystallization protocol, the effect of the PLLA semicrystalline matrix in the crystallization of the PCL block was evaluated (see Figure 8). 
The second crystallization protocol (protocol 2) will be described after the results obtained with the first one are analyzed.

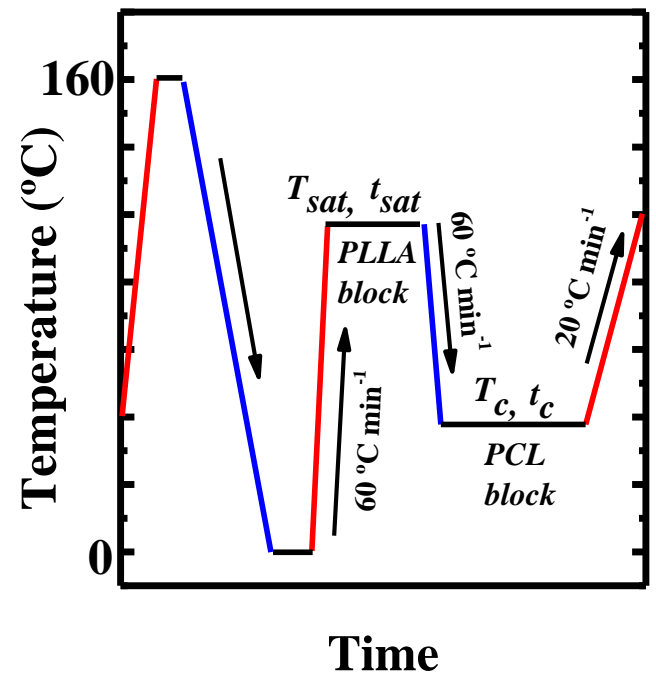

Figure 8. Two step crystallization protocol (protocol 1) employ to follow the isothermal crystallization of the PCL block with the PLLA phase previously crystallized.

The inverse half-crystallization time $\left(1 / \tau_{50} \%\right)$ values of the PCL homopolymer and the PCL block in the diblock copolymers and terpolymers are depicted in Figure 9. Since the PCL block crystallization was followed after quenching from a molten PCL phase, the $1 / \tau_{50 \%}$ values correspond to the right side of the bell-shape crystallization rate curve (low supercoolings). The previously formed PLLA crystals had a nucleating effect on the crystallization of the PCL chains. Therefore, the supercooling needed to crystallize the PCL block decreased. Also, the crystallization kinetics of the PCL block in both terpolymers and diblock copolymers increased, in comparison to the PCL homopolymer. Despite the PCL block had to crystallize inside the interlamellar regions of the PLLA spherulitic 
superstructure, this fact did not perturbed the PCL crystallization. This is an important observation in these terpolymers. The order of the blocks in the terpolymers will affect the crystallization kinetics. Since, the middle PCL block had one end attached to the PLLA crystals and the other to a molten PEO chain, the PLLA crystals played as successful nucleating sites for the crystallization of the PCL chains and therefore their crystallization kinetics was enhanced. In a previous publication about PCL-b-PLLA diblock copolymers, ${ }^{36}$ some of us reported a hampered crystallization of the PCL block when the PLLA block was previously crystallized until saturation. Even thought a nucleating effect of the PLLA crystals was demonstrated by self-nucleation experiments, larger supercoolings were needed to crystallize the PCL block and a retarded overall crystallization kinetics was observed. Two of those diblock copolymers had a similar PCL content (40 and $56 \%$ ) to that of the copolymers reported here (43 and $59 \%$ ). However, the length of the PCL block is different. Besides the nucleating effect of the PLLA crystals, the increase in the crystallization rate shown in Figure 9 obeyed to the smaller PCL block length. The molecular weight of the PCL homopolymer and PCL block in the copolymers and terpolymers was 7000, 6600 and 6800 $\mathrm{g} \mathrm{mol}^{-1}$, respectively. In contrast, the molecular weight of the PCL block in the diblock copolymers reported previously was 8500 and $14200 \mathrm{~g} \mathrm{~mol}^{-1} \cdot{ }^{36}$

At higher PLLA content, the crystallization trend of the PCL block in the terpolymer and the diblock copolymer was the same (PCL43 $\mathrm{PLLA}_{57}{ }^{15.4}$ and $\mathrm{PEO}_{23} \mathrm{PCL}_{34} \mathrm{PLLA}_{43}{ }^{19.9}$ samples). As the PLLA content reduces (PCL content increases), the crystallization kinetics of the PCL block in the $\mathrm{PEO}_{29} \mathrm{PCL}_{42} \mathrm{PLLA}_{29}{ }^{16.1}$ terpolymer was only slightly higher than in the PCL 59 PLLA $41^{11.2}$ diblock copolymer (see Figure 9, left). Therefore, the dominant effect is the nucleation induced by the previously formed PLLA crystals. 

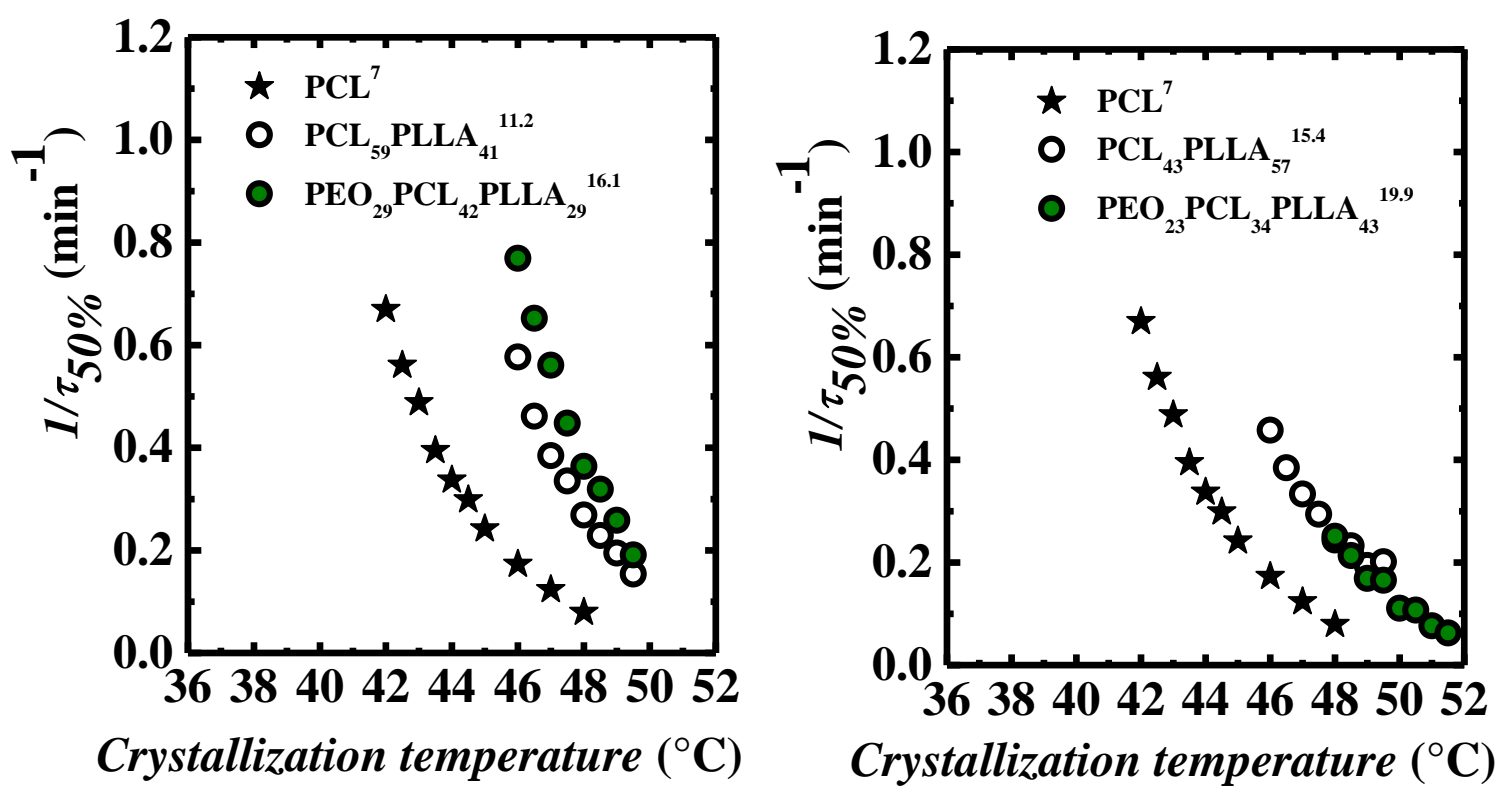

Figure 9. Inverse of half crystallization times $\left(1 / \tau_{50 \%}\right)$ versus crystallization temperature of the PCL homopolymer and the PCL block within the diblock copolymers and terpolymers, after the PLLA block was first isothermally crystallized until saturation.

Additionally, the content of PCL did not affect considerably the crystallization kinetics of the PCL block. Only a slight increment in the $1 / \tau 50 \%$ values was observed as the PCL content in the terpolymers increased. The molecular weight of the PCL block is not to take into consideration, since the length values are almost the same as in the PCL homopolymer (7000 $\mathrm{g} \mathrm{mol}^{-1}$ of the PCL homopolymer, and 6600 and $6800 \mathrm{~g} \mathrm{~mol}^{-1}$, of the PCL block in both copolymers and terpolymers, respectively).

Despite the enhanced crystallization kinetics, the crystallinity degree of the PCL block in the diblock copolymers reduced, in comparison to the PCL homopolymer (see Figure 10). As expected, the crystallinity was even lower as the PCL content in the diblock copolymer reduces (see sample PCL43 PLLA $_{57}{ }^{15.4}$ versus PCL $_{59}$PLLA $_{41}{ }^{11.2}$ in Figure 10). Since, the PCL block had to crystallize inside the interlamellar regions of the PLLA 
crystalline superstructure, the fraction of amorphous PCL phase that could convert into crystals was highly diminished. The previously formed PLLA lamellar crystals within the template spherulitic superstructure restricted the PCL block lamellae to grow in between the lamellar stacks of the PLLA.
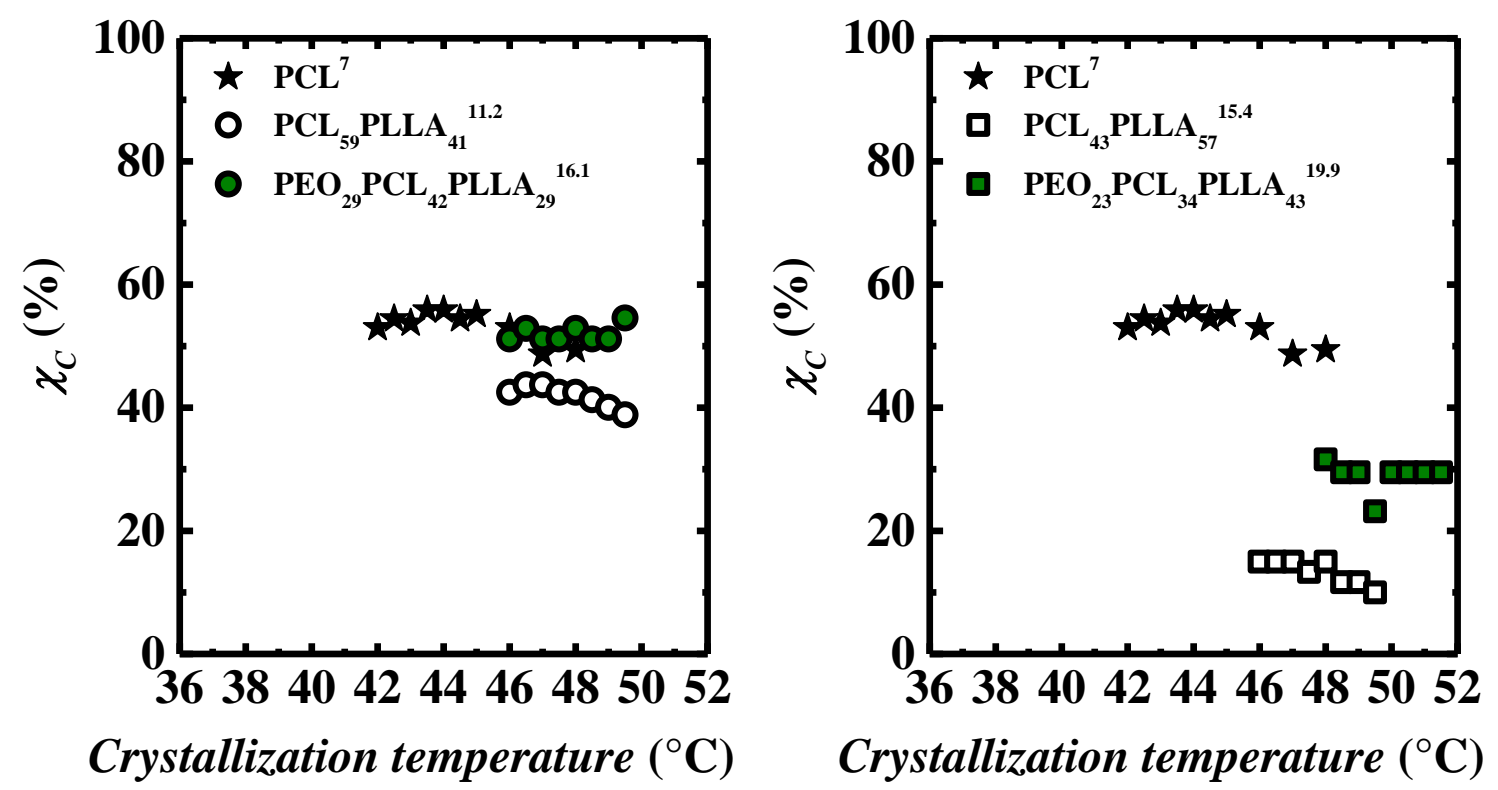

Figure 10. Crystallization degree $\left(\chi_{c}\right)$ of the PCL block within the diblock copolymers and terpolymers indicated, after first isothermally crystallizing the PLLA block until saturation.

On the contrary, the presence of the molten PEO block in the terpolymer contributed to increase the crystallinity of the PCL block; in comparison to the diblock copolymers (see samples $\mathrm{PEO}_{29} \mathrm{PCL}_{42} \mathrm{PLLA}_{29}{ }^{16.1}$ and $\mathrm{PEO}_{23} \mathrm{PCL}_{34} \mathrm{PLLA}_{43}{ }^{19.9}$ in Figure 10). In fact, the PCL crystallinity degree in the terpolymer with larger PCL content even matched that of the PCL homopolymer (see Figure 10, left). In both terpolymers, the amorphous PEO chains enhanced the crystallizability of the PCL block, increasing the mobility and diffusion of the PCL chains (which are anchored to the PLLA crystals) to the growing PCL crystal front. 
The Avrami fitting was also applied to the experimental data registered during the isothermal crystallization of the PCL homopolymer and the PCL block in the diblock copolymers and terpolymers. The Avrami index values of all the samples were between 2 and 3 (results no shown, see Figure S2 in Supporting Information).

The crystallization of the PCL block in the terpolymers and block copolymers was also followed employing a one step protocol (protocol2). The method consisted in quenching the sample from the melt until the crystallization temperature of the PCL block. Then, the PCL isothermal crystallization was registered at that temperature, and finally, the sample was heated again until its melting (see Figure 11). Again, in the case of the terpolymers, the crystallization temperatures chosen were high enough to ensure that only the PCL block was crystallizing, while the PEO block remained molten. In addition, during the cooling scan the PLLA block did not crystallized. A cold crystallization of the PLLA block took place during the subsequent heating scan, after the melting of the isothermally crystallized PCL crystals. Unlike the two step protocol, in this method the PCL block crystallization was followed while the PLLA phase remained amorphous.

The isothermal crystallization kinetics of the PCL block, surrounded by an amorphous PLLA phase in the case of the diblock or an amorphous PLLA and PEO phase, in the case of the triblock, is shown in Figure 12. Comparing the $1 / \tau_{50 \%}$ values to those plotted in Figure 9 , it is clear that the crystallization behavior was completely different under this condition. In comparison to the PCL homopolymer, the crystallization rate of the PCL block in the diblock copolymers was largely decreased. 


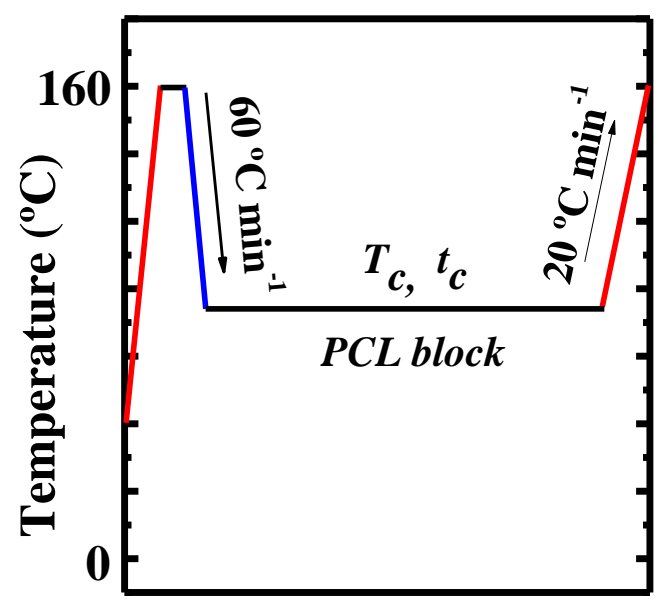

Time

Figure 11. One step crystallization protocol (protocol 2) to follow the crystallization of the PCL block while keeping the PLLA phase amorphous.

While previously formed PLLA crystals caused a nucleating effect (when protocol 1 was employed), in this case the amorphous PLLA phase induced an anti-plasticizing effect. The PCL block required a larger supercooling in order to crystallize, and the overall crystallization kinetics greatly reduces. These PCL- $b$-PLLA diblock copolymers have been reported to be miscible or weakly segregated in the melt. When the sample is quenched from the melt until the PCL crystallization temperature, chains of the PCL and PLLA blocks are mixed together. In order to crystallize, the PCL chains had to demix from this amorphous phase that contains more rigid PLLA chains, and have to come closer to form a nucleus or to grow in the crystalline front. Therefore, under this condition, the phase separation is driven by the enthalpy of demixing of the constituent blocks during the crystallization of the PCL block. Since this is a miscible system and the glass transition of PLLA is higher than that of PCL, the glass transition in the diblock copolymer probably takes place at higher temperature 
than in the PCL homopolymer. Therefore, the PCL is probably crystallizing at temperatures that are closer to $T_{g}$. As a result, the mobility of the PCL chains should have reduced considerably. In addition, at temperatures in which PCL crystallizes, the chain movements of the more rigid PLLA block had also slowed down, making more difficult the diffusion and crystallization of the PCL chains covalently attached to them.
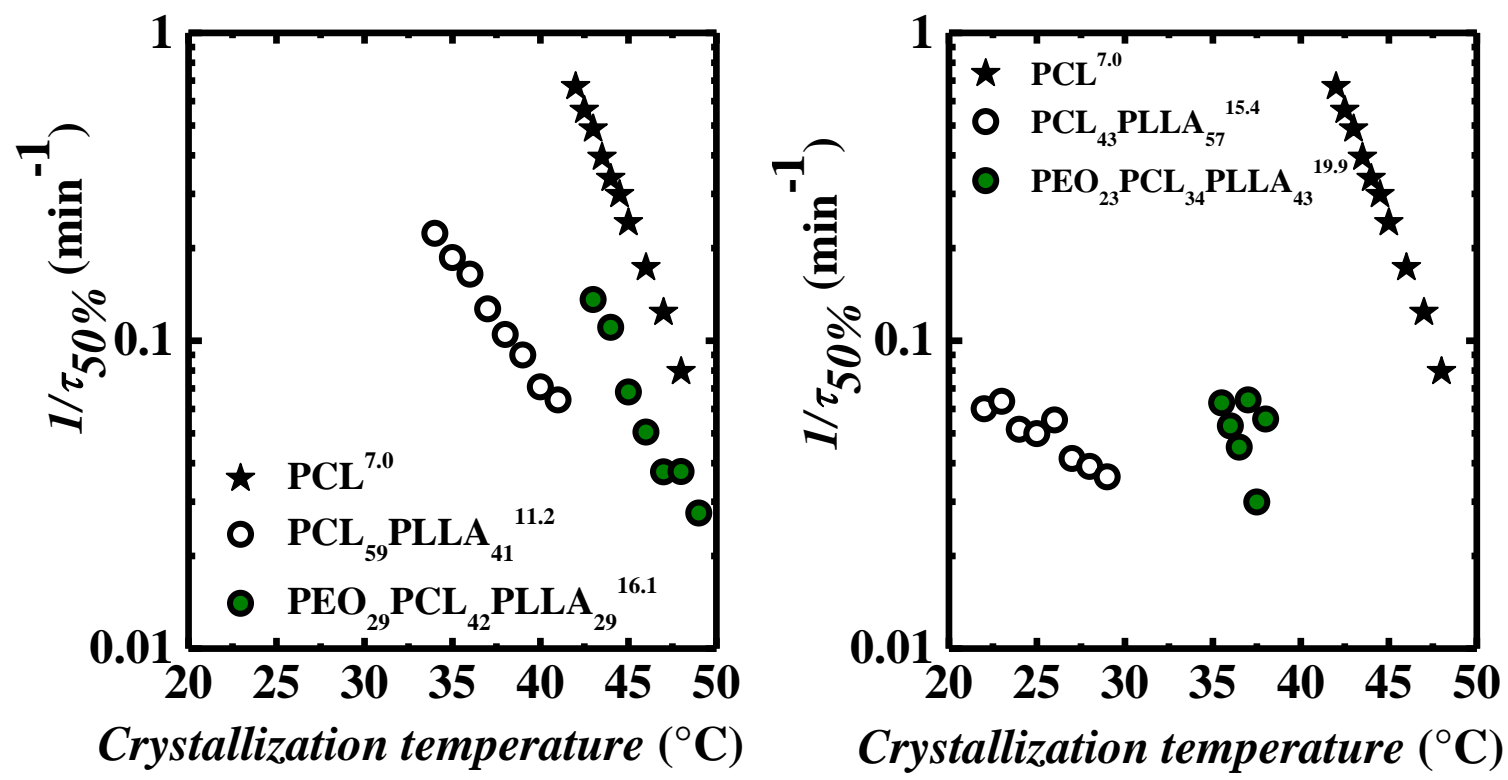

Figure 12. Inverse of half crystallization times $\left(1 / \tau_{50 \%}\right)$ versus crystallization temperature of the PCL homopolymer and the PCL block within the diblock copolymers and terpolymers, crystallized in one step (protocol 2).

In contrast, when a third block of PEO is attached to PCL- $b$-PLLA copolymers, the crystallization behavior of the middle PCL block became very interesting. From Figure 12 it is clear that the overall crystallization rate and crystallization temperature of the PCL block in the terpolymers increased, in comparison to the same PCL block in the analogous diblock copolymers. The middle PCL block crystallized under smaller supercooling in both terpolymers. The PCL crystallizes at temperatures where the PEO block remains molten. As 
in the two step crystallization protocol (protocol 1), the molten PEO chains enhanced the crystallization ability of the PCL block due to a plasticizing effect. In the terpolymers, the PCL crystallized from a mixed amorphous phase that includes more rigid amorphous chains of PLLA as well as molten PCL and PEO chains. As the crystallization took place, the molten PEO chains increased the mobility and diffusion of the PCL chains to the crystalline front. However and as expected, the $1 / \tau 50 \%$ values did not match those of the PCL homopolymer, even though the crystallization rate of the PCL block in the terpolymers was increased. In the PCL homopolymer, the chains can form nucleus and grow freely from the melt. While in the terpolymers, the PCL chains are covalently attached to more rigid PLLA chains in the melt state that would hinder the ability of the PCL block to undergo crystallization.

Unlike the two step crystallization protocol (protocol 1), the composition affected the crystallization kinetics of the PCL block in the terpolymers and diblock copolymers when they were crystallized in one step (protocol 2). By extrapolating the $1 / \tau_{50} \%$ values to higher crystallization temperatures in Figure 12, right, it is clear that the crystallization rate became faster as the PCL content increases, when comparing both terpolymers and diblock copolymers separately.

Despite the enhanced crystallization kinetics due to the presence of the molten PEO block, the crystallinity degree of the PCL block in the diblock copolymer and the terpolymer reduced or remained almost constant at the supercooling evaluated (see Figure S3 in the Supporting Information). In addition, the Avrami index values of all the samples were between 2 and 3 (see Figure S4 in Supporting Information).

\section{Overall crystallization kinetics of the PEO block in the terpolymers}


Following the crystallization kinetics of the third PEO block was extremely difficult since the crystallization and melting temperatures of both PEO and PCL are very similar. A three step crystallization protocol was the procedure employed to evaluate the crystallization behavior. The PLLA block was first crystallized until saturation, and next, the sample was quenched until the crystallization temperature of the PCL block. Then, the PCL block was crystallized until saturation, while the PEO chains remained molten. Finally, the sample was quenched again until the PEO crystallization temperature was reached and the isothermal crystallization was recorded (see Figure 13). The purpose of this protocol was to evaluate the effect of the double crystalline PCL/PLLA lamellae formed previously in the subsequent PEO block crystallization. The isothermal crystallization of the PEO block in the terpolymers was compared to the analogous PEO homopolymer. Attempts were made to register the crystallization behavior of the PEO block within analogous PEO- $b$-PCL diblock copolymers. However, due to the similarity between the crystallization temperatures of both blocks, it was not possible to isolate the crystallization of one block from the other. In other words, both blocks crystallized simultaneously.

In Figure 14 are depicted the half crystallization times $\left(1 / \tau_{50 \%}\right)$ of the PEO block in the $\mathrm{PEO}_{29} \mathrm{PCL}_{42} \mathrm{PLLA}_{29}{ }^{16.1}$ triblock terpolymer and the PEO homopolymer. It was not possible to isolate the PEO crystallization from that of the PCL block in the other $\mathrm{PEO}_{23} \mathrm{PCL}_{34} \mathrm{PLLA}_{43}{ }^{19.9}$ terpolymer sample, after the crystallization of the PCL and the PLLA blocks. In this particular sample, very high isothermal crystallization temperatures (only 10 degrees lower than the crystallization temperature used to crystallize the PCL block until saturation) were needed in order to avoid crystallization during the cooling to Tc. However, 
at such high temperatures, the crystallization times are very long, and the heat flow developed as function of time is so small that it was not possible to detect by the DSC equipment.

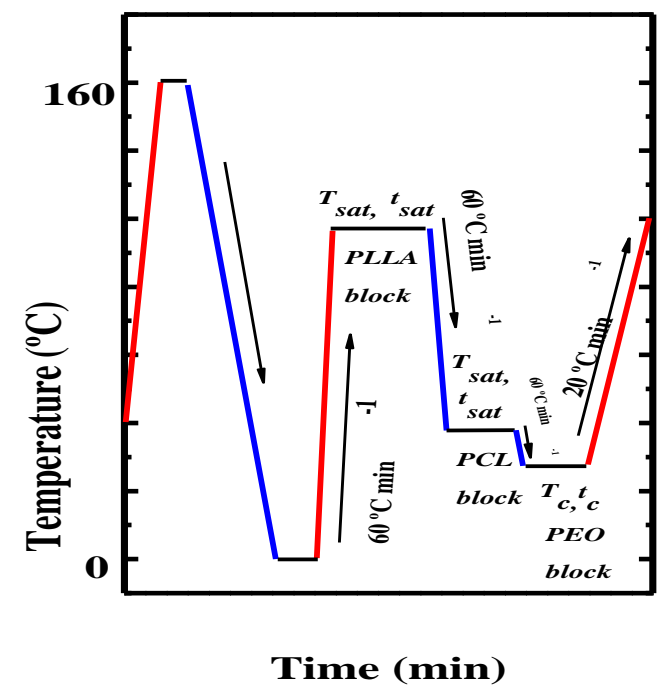

Figure 13. Three step crystallization protocol to follow the crystallization of the PCL block with the PLLA phase previously crystallized.

The PEO block crystallization rate in the $\mathrm{PEO}_{29} \mathrm{PCL}_{42} \mathrm{PLLA}_{29}{ }^{16.1}$ terpolymer highly decreased in comparison to the PEO homopolymer, as well as the crystallization temperature. A higher supercooling was needed to crystallize the PEO block. In these triblock terpolymers, the PEO block is an end block and therefore the PEO chains are attached to PCL crystals at one end but free at the other end. Despite of that, the previously formed PLLA and PCL rigid crystals created a hard environment that highly confined the crystallization of the PEO block. In another publication, ${ }^{68}$ we reported the theoretical analysis of the SAXS curves of the triblock terpolymer employing one-dimensional structural models. The modeling suggested that either a lamella of PCL or a lamella of PEO inserted randomly between two adjacent 
PLLA lamellae, but not both PCL and PEO blocks together. The PEO molten chains (located at the end of the terpolymer) are covalently bonded to the PCL block in the middle. Therefore, the PEO chains had no other choice but to crystallize inside the interlamellar spaces left in between PLLA crystalline lamellae. Thus, the confinement imposed by the previously formed lamellar crystals will hinder PEO crystallization. ${ }^{24}$ As a result, the crystallinity degree of the PEO block in the terpolymer reduced (see Figure S5 in the Supporting Information) Evidences of confinement have been reported in PLLA- $b$-PEO block copolymers with short PEO block length or high PLLA content, in which the PLLA block crystallized first. ${ }^{40,69}$ Despite the hindered crystallization of the PEO block, the Avrami index did not fit behavior observed, since the index values remained similar to those of the PEO homopolymer (around 2) (see Figure S6 in the Supporting Information).

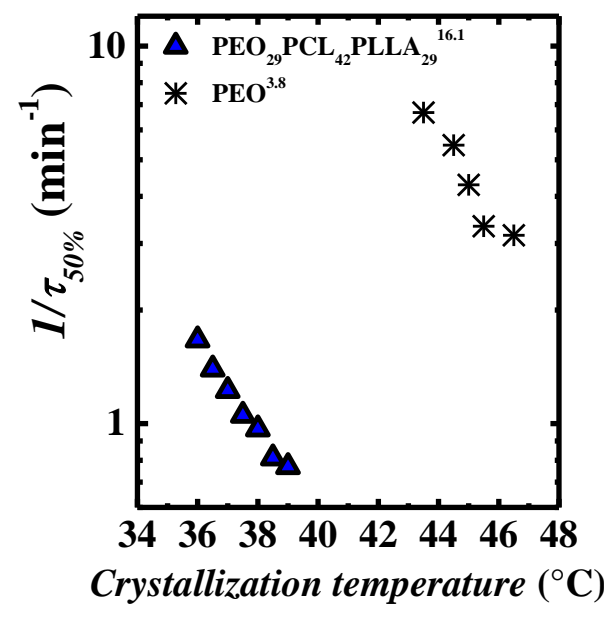

Figure 14. Inverse of half crystallization times $\left(1 / \tau_{50} \%\right)$ as function of crystallization temperature for the PEO homopolymer and the PEO block within the $\mathrm{PEO}_{29} \mathrm{PCL}_{42} \mathrm{PLLA}_{29}{ }^{16.1}$ triblock terpolymer.

CONCLUSIONS 
The overall isothermal crystallization of each block in PEO-b-PCL-b-PLLA triblock terpolymers was studied by DSC, and compared to the analogous diblock copolymers and homopolymers of identical block length to those in the terpolymers. The PLLA crystallization was followed at a temperature in which the PCL and PEO chains were molten. The molten chains in the terpolymers caused a strong plasticizing effect (higher supercooling) on the PLLA crystallization, as compared to PLLA block in the diblock copolymers. The effect was more notorious as the PLLA content in the terpolymer was reduced. Surprisingly, the overall crystallization rate of PLLA block was higher in both terpolymers in comparison to the diblock copolymers, but the crystallization degree reduced.

The previously formed PLLA crystals had a nucleating effect on the PCL crystallization, and therefore, increased crystallization rate and decreased supercooling were observed. In addition, the PEO molten chains contributed to enhance the PCL crystallization. However, the PCL block in the terpolymers exhibited a reduced crystallinity degree due to the restriction imposed by the PLLA crystals. On the contrary, when the PCL block is crystallized without previously crystallizing the PLLA block, the PLLA amorphous phase caused anti-plasticizing effect on PCL crystallization. The overall crystallization kinetics greatly reduces. However, the presence of the PEO block in the terpolymers enhanced the overall crystallization rate the PCL block, in comparison to the diblock copolymers. Following the crystallization of the PEO block after PLLA and PCL fully crystallized was extremely difficult, since relatively high crystallization temperatures were required to avoid PEO crystallization during quenching. The crystallization rate highly decreased and the supercooling increased as a result of the confinement imposed by the previously formed PLLA and PCL crystals. 
The crystallization behavior of PEO- $b$-PCL- $b$-PLLA triblock terpolymers with three crystallizable phases relies on the complex interplay between the different blocks and the crystallization conditions. Whether one of the phases is molten, amorphous or crystalline, it would affect the ability to crystallize of the others two, and as a result, complex effects such as plasticizing, nucleation, anti-plasticizing and confinement might take place. The addition of a third crystallizable block would definitely broaden the potential features of these terpolymers for particular applications as biodegradable materials. Therefore, understanding the crystallization behavior of triple crystalline triblock terpolymers is expected to be in the focus of researchers for the next years.

\section{ACKNOWLEDGEMENTS}

The authors would like to acknowledge funding by ALBA project 2016091863 (2017). We would also like to acknowledge staff from the BL11 beamline at ALBA synchrotron, as well as travel funding by "Ministerio de Economía y Competitividad" and "Generalidad de Cataluña" (Grant Number: 2016091863). We gratefully acknowledge funds received by “Mineco MAT2014-53437-C2-P”. We are also grateful to Dr. Eva Crosas at ALBA Synchrotron, Cerdanyola del Vallès, Barcelona, Spain and Dr. Daniel Fulla at Deutsches Elektronen-Synchrotron DESY, Hamburg, Germany; for their collaboration with the SAXS

data treatment. Dr. Eva Crosas was supported by the Spanish Nuclear Safety Council (CSN).

\section{SUPPORTING INFORMATION}


Additional DSC scans, crystallization degree and Avrami Index values of the diblock copolymers and terpolymers crystallized using different protocols.

\section{REFERENCES}

(1) Hamley, I. W., The Physics of Block Copolymers. Oxford University Press: Oxford 1998.

(2) Huang, S.; Jiang, S. Structures and Morphologies of Biocompatible and Biodegradable Block Copolymers. RSC Advances 2014, 4, 24566-24583.

(3) Müller, A. J.; Arnal, M. L.; Lorenzo, A. T. In Handbook of Polymer Crystallization, Piorkowska, E.,Rutledge, G. C., Eds.; John Wiley and Sons: Hoboken, New Jersey, 2013; p 347.

(4) Li, S.; Register, R. A. In Handbook of Polymer Crystallization, Piorkowska, E.,Rutledge, G. C., Eds.; John Wiley and Sons: Hoboken, New Jersey, 2013; p 327.

(5) He, W. N.; Xu, J. T. Crystallization Assisted Self-Assembly of Semicrystalline Block Copolymers. Prog. Polym. Sci. 2012, 37, 1350-1400.

(6) Castillo, R. V.; Müller, A. J. Crystallization and Morphology of Biodegradable or Biostable Single and Double Crystalline Block Copolymers. Prog. Polym. Sci. 2009, 34, 516560.

(7) Nakagawa, S.; Marubayashi, H.; Nojima, S. Crystallization of Polymer Chains Confined in Nanodomains. Eur. Polym. J. 2015, 70, 262-275.

(8) Michell, R. M.; Müller, A. J. Confined Crystallization of Polymeric Materials. Prog. Polym. Sci. 2016, 54-55, 183-216.

(9) Müller, A. J.; Arnal, M. L.; Balsamo, V. Crystallization in Block Copolymers with More Than One Crystallizable Block. Lecture Notes in Physics 2007, 714, 229-259. 
(10) Müller, A. J.; Balsamo, V.; Arnal, M. L. Nucleation and Crystallization in Diblock and Triblock Copolymers. Adv. Polym. Sci. 2005, 190, 1-63.

(11) Abetz, V.; Simon, P. F. W. Phase Behaviour and Morphologies of Block Copolymers. Adv. Polym. Sci. 2005, 189, 125-212.

(12) Hamley, I. Crystallization in Block Copolymers. Adv. Polym. Sci. 1999, 148, 113-137.

(13) Hamley, I. W. In Encyclopedia of Polymer Science and Technology, John Wiley \& Sons, Inc.: 2002.

(14) Hadjichristidis, N.; Pitsikalis, M.; Iatrou, H. Synthesis of Block Copolymers. Advances in Polymer Science 2005, 189, 1-124.

(15) Loo, Y. L.; Register, R. A.; Ryan, A. J. Modes of Crystallization in Block Copolymer Microdomains: Breakout, Templated, and Confined. Macromolecules 2002, 35, 2365-2374. (16) Boschetti-De-Fierro, A.; Fierro, D.; Albuerne, J.; Funari, S. S.; Abetz, V. Thermal Monitoring of Morphology in Triblock Terpolymers with Crystallizable Blocks. J. Polym. Sci., Part B: Polym. Phys. 2007, 45, 3197-3206.

(17) Müller, A. J.; Albuerne, J.; Marquez, L.; Raquez, J. M.; Degée, P.; Dubois, P.; Hobbs, J.; Hamley, I. W. Self-Nucleation and Crystallization Kinetics of Double Crystalline Poly( $\rho-$ Dioxanone)-b-Poly(ع-Caprolactone) Diblock Copolymers. Faraday Discuss. 2005, 128, 231252.

(18) Müller, A. J.; Albuerne, J.; Esteves, L. M.; Marquez, L.; Raquez, J. M.; Degée, P.; Dubois, P.; Collins, S.; Hamley, I. W. Confinement Effects on the Crystallization Kinetics and Self-Nucleation of Double Crystalline Poly( $\rho$-Dioxanone)-b-Poly( $\varepsilon$-Caprolactone) Diblock Copolymers. Macromol. Sym. 2004, 215, 369-382. 
(19) Albuerne, J.; Márquez, L.; Müller, A. J.; Raquez, J. M.; Degée, P.; Dubois, P.; Castelletto, V.; Hamley, I. W. Nucleation and Crystallization in Double Crystalline Poly( $\rho-$ Dioxanone)-b-Poly( $\varepsilon$-Caprolactone) Diblock Copolymers. Macromolecules 2003, 36, 16331644.

(20) Arnal, M. L.; Balsamo, V.; López-Carrasquero, F.; Contreras, J.; Carrillo, M.; Schmalz, H.; Abetz, V.; Laredo, E.; Müller, A. J. Synthesis and Characterization of Polystyrene- $b$ Poly(Ethylene Oxide)-b-Poly( $\varepsilon$-Caprolactone) Block Copolymers. Macromolecules 2001, $34,7973-7982$.

(21) Balsamo, V.; Paolini, Y.; Ronca, G.; Müller, A. J. Crystallization of the Polyethylene Block in Polystyrene- $b$-Polyethylene- $b$-Polycaprolactone Triblock Copolymers, 1: SelfNucleation Behavior. Macromol. Chem. Phys. 2000, 201, 2711-2720.

(22) Balsamo, V.; Müller, A. J.; Stadler, R. Antinucleation Effect of the Polyethylene Block on the Polycaprolactone Block in Abc Triblock Copolymers. Macromolecules 1998, 31, 7756-7763.

(23) Balsamo, V.; Müller, A. J.; Von Gyldenfeldt, F.; Stadler, R. Ternary Abc Block Copolymers Based on One Glassy and Two Crystallizable Blocks: Polystyrene-BlockPolyethylene-Block-Poly(E-Caprolactone). Macromol. Chem. Phys. 1998, 199, 1063-1070.

(24) Palacios, J. K.; Mugica, A.; Zubitur, M.; Iturrospe, A.; Arbe, A.; Liu, G.; Wang, D.; Zhao, J.; Hadjichristidis, N.; Muller, A. J. Sequential Crystallization and Morphology of Triple Crystalline Biodegradable PEO- $b$-PCL- $b$-PLLA Triblock Terpolymers. $R S C$ Advances 2016, 6, 4739-4750.

(25) Sun, L.; Shen, L. J.; Zhu, M. Q.; Dong, C. M.; Wei, Y. Synthesis, Self-Assembly, DrugRelease Behavior, and Cytotoxicity of Triblock and Pentablock Copolymers Composed of 
Poly(E-Caprolactone), Poly(L-Lactide), and Poly(Ethylene Glycol). Journal of Polymer Science Part A: Polymer Chemistry 2010, 48, 4583-4593.

(26) Chiang, Y.-W.; Hu, Y.-Y.; Li, J.-N.; Huang, S.-H.; Kuo, S.-W. Trilayered Single Crystals with Epitaxial Growth in Poly(Ethylene Oxide)-Block-Poly( $\varepsilon$-Caprolactone)Block-Poly(L-Lactide) Thin Films. Macromolecules 2015, 48, 8526-8533.

(27) Cai, C.; Wang, L. U.; Donc, C. M. Synthesis, Characterization, Effect of Architecture on Crystallization, and Spherulitic Growth of Poly(L-Lactide)- $b$-Poly(Ethylene Oxide) Copolymers with Different Branch Arms. J. Polym. Sci., Part A: Polym. Chem. 2006, 44, 2034-2044.

(28) Vainionpää, S.; Rokkanen, P.; Törmälä, P. Surgical Applications of Biodegradable Polymers in Human Tissues. Progress in Polymer Science 1989, 14, 679-716.

(29) Ho, R. M.; Hsieh, P. Y.; Tseng, W. H.; Lin, C. C.; Huang, B. H.; Lotz, B. CrystallizationInduced Orientation for Microstructures of Poly(L-Lactide)- $b$-Poly( $\varepsilon$-Caprolactone) Diblock Copolymers. Macromolecules 2003, 36, 9085-9092.

(30) Jeon, O.; Lee, S. H.; Kim, S. H.; Lee, Y. M.; Kim, Y. H. Synthesis and Characterization of Poly(L-Lactide)-Poly( $\varepsilon$-Caprolactone) Multiblock Copolymers. Macromolecules 2003, $36,5585-5592$.

(31) Hamley, I. W.; Parras, P.; Castelletto, V.; Castillo, R. V.; Müller, A. J.; Pollet, E.; Dubois, P.; Martin, C. M. Melt Structure and Its Transformation by Sequential Crystallization of the Two Blocks within Poly(L-Lactide)-Block-Poly( $\varepsilon$-Caprolactone) Double Crystalline Diblock Copolymers. Macromolecular Chemistry and Physics 2006, 207, 941-953.

(32) Hamley, I. W.; Castelletto, V.; Castillo, R. V.; Müller, A. J.; Martin, C. M.; Pollet, E.; Dubois, P. Crystallization in Poly(L-Lactide)-b-Poly( $\varepsilon$-Caprolactone) Double Crystalline 
Diblock Copolymers: A Study Using X-Ray Scattering, Differential Scanning Calorimetry, and Polarized Optical Microscopy. Macromolecules 2005, 38, 463-472.

(33) Wang, J. L.; Dong, C. M. Synthesis, Sequential Crystallization and Morphological Evolution of Well-Defined Star-Shaped Poly( $\varepsilon$-Caprolactone)- $b$-Poly(L-Lactide) Block Copolymer. Macromol. Chem. Phys. 2006, 207, 554-562.

(34) Yan, D.; Huang, H.; He, T.; Zhang, F. Coupling of Microphase Separation and Dewetting in Weakly Segregated Diblock Co-Polymer Ultrathin Films. Langmuir 2011, 27, 11973-11980.

(35) Peponi, L.; Navarro-Baena, I.; Báez, J. E.; Kenny, J. M.; Marcos-Fernández, A. Effect of the Molecular Weight on the Crystallinity of PCL- $b$-PLLA Di-Block Copolymers. Polymer 2012, 53, 4561-4568.

(36) Castillo, R. V.; Müller, A. J.; Raquez, J. M.; Dubois, P. Crystallization Kinetics and Morphology of Biodegradable Double Crystalline PLLA- $b$-PCL Diblock Copolymers. Macromolecules 2010, 43, 4149-4160.

(37) Laredo, E.; Prutsky, N.; Bello, A.; Grimau, M.; Castillo, R. V.; Müller, A. J.; Dubois, P. Miscibility in Poly(L-Lactide)- $b$-Poly( $\varepsilon$-Caprolactone) Double Crystalline Diblock Copolymers. Eur. Phys. J. E 2007, 23, 295-303.

(38) Casas, M. T.; Puiggalí, J.; Raquez, J. M.; Dubois, P.; Córdova, M. E.; Müller, A. J. Single Crystals Morphology of Biodegradable Double Crystalline PLLA- $b$-PCL Diblock Copolymers. Polymer 2011, 52, 5166-5177.

(39) Barthel, M. J.; Schacher, F. H.; Schubert, U. S. Poly(Ethylene Oxide) (PEO)-Based ABC Triblock Terpolymers-Synthetic Complexity Vs. Application Benefits. Polym. Chem. 2014, $5,2647-2662$. 
(40) Yang, J.; Zhao, T.; Cui, J.; Liu, L.; Zhou, Y.; Li, G.; Zhou, E.; Chen, X. Nonisothermal Crystallization Behavior of the Poly(Ethylene Glycol) Block in Poly(L-Lactide)Poly(Ethylene Glycol) Diblock Copolymers: Effect of the Poly(L-Lactide) Block Length. $J$. Polym. Sci., Part B: Polym. Phys. 2006, 44, 3215-3226.

(41) Huang, S.; Jiang, S.; An, L.; Chen, X. Crystallization and Morphology of Poly(Ethylene Oxide- $b$-Lactide) Crystalline-Crystalline Diblock Copolymers. J. Polym. Sci., Part B: Polym. Phys. 2008, 46, 1400-1411.

(42) Huang, S.; Li, H.; Jiang, S.; Chen, X.; An, L. Morphologies and Structures in Poly(LLactide- $b$-Ethylene Oxide) Copolymers Determined by Crystallization, Microphase Separation, and Vitrification. Polym. Bull. 2011, 67, 885-902.

(43) Shin, D.; Shin, K.; Aamer, K. A.; Tew, G. N.; Russell, T. P.; Lee, J. H.; Jho, J. Y. A Morphological Study of a Semicrystalline Poly(L-Lactic Acid- $b$-Ethylene Oxide- $b$-L-Lactic Acid) Triblock Copolymer. Macromolecules 2005, 38, 104-109.

(44) Xue, F.; Chen, X.; An, L.; Funari, S. S.; Jiang, S. Crystallization Induced Layer-to-Layer Transitions in Symmetric PEO- $b$-PLLA Block Copolymer with Synchrotron Simultaneous Saxs/Waxs Investigations. RSC Advances 2014, 4, 56346-56354.

(45) Huang, C. I.; Tsai, S. H.; Chen, C. M. Isothermal Crystallization Behavior of Poly(LLactide) in Poly(L-Lactide)-Block-Poly(Ethylene Glycol) Diblock Copolymers. Journal of Polymer Science Part B: Polymer Physics 2006, 44, 2438-2448.

(46) Kim, K. S.; Chung, S.; Chin, I. J.; Kim, M. N.; Yoon, J. S. Crystallization Behavior of Biodegradable Amphiphilic Poly(Ethylene Glycol)-Poly(L-Lactide) Block Copolymers. J. Appl. Polym. Sci. 1999, 72, 341-348. 
(47) Yang, J.; Liang, Y.; Han, C. C. Effect of Crystallization Temperature on the Interactive Crystallization Behavior of Poly(L-Lactide)-Block-Poly(Ethylene Glycol) Copolymer. Polymer (United Kingdom) 2015, 79, 56-64.

(48) Zhou, D.; Sun, J.; Shao, J.; Bian, X.; Huang, S.; Li, G.; Chen, X. Unusual Crystallization and Melting Behavior Induced by Microphase Separation in MPEG- $b$-PLLA Diblock Copolymer. Polymer 2015, 80, 123-129.

(49) Guillerm, B.; Lemaur, V.; Ernould, B.; Cornil, J.; Lazzaroni, R.; Gohy, J. F.; Dubois, P.; Coulembier, O. A One-Pot Two-Step Efficient Metal-Free Process for the Generation of PEO-b-PCL-b-PLA Amphiphilic Triblock Copolymers. RSC Advances 2014, 4, 1002810038.

(50) Zhao, J.; Pahovnik, D.; Gnanou, Y.; Hadjichristidis, N. Sequential Polymerization of Ethylene Oxide, E-Caprolactone and L-Lactide: A One-Pot Metal-Free Route to Tri- and Pentablock Terpolymers. Polymer Chemistry 2014, 5, 3750-3753.

(51) Alamri, H.; Zhao, J.; Pahovnik, D.; Hadjichristidis, N. Phosphazene-Catalyzed RingOpening Polymerization of $\varepsilon$-Caprolactone: Influence of Solvents and Initiators. Polymer Chemistry 2014, 5, 5471-5478.

(52) Lorenzo, A. T.; Arnal, M. L.; Albuerne, J.; Müller, A. J. Dsc Isothermal Polymer Crystallization Kinetics Measurements and the Use of the Avrami Equation to Fit the Data: Guidelines to Avoid Common Problems. Polym. Test. 2007, 26, 222-231.

(53) Androsch, R.; Di Lorenzo, M. L. Crystal Nucleation in Glassy Poly(L-Lactic Acid). Macromolecules 2013, 46, 6048-6056. 
(54) Müller, A. J.; Avila, M.; Saenz, G.; Salazar, J. In Poly(Lactic Acid) Science and Technology: Processing, Properties, Additives and Applications, Jimenez, A.,Peltzer, M.,Ruseckaite, R., Eds.; The Royal Society of Chemistry: Cambridge, 2015.

(55) Gedde, U. W., Polymer Physics. Chapman \& Hall: London, 1995.

(56) Kim, J. K.; Park, D.-J.; Lee, M.-S.; Ihn, K. J. Synthesis and Crystallization Behavior of Poly(L-Lactide)-Block-Poly(ع-Caprolactone) Copolymer. Polymer 2001, 42, 7429-7441.

(57) Pan, P.; Kai, W.; Zhu, B.; Dong, T.; Inoue, Y. Polymorphous Crystallization and Multiple Melting Behavior of Poly(L-Lactide): Molecular Weight Dependence. Macromolecules 2007, 40, 6898-6905.

(58) Mandelkern, L., Crystallization of Polymers: Volume 2, Kinetics and Mechanisms. Cambridge University Press: 2004.

(59) Göler, F. v.; Sachs, G. Zur Kinetik Von Kristallisationsvorgängen. Physik 1932, 77, 281286.

(60) Ergoz, E.; Fatou, J. G.; Mandelkern, L. Molecular Weight Dependence of the Crystallization Kinetics of Linear Polyethylene. I. Experimental Results. Macromolecules 1972, 5, 147-157.

(61) Müller, A. J.; Michell, R. M.; Lorenzo, A. T. In Polymer Morphology: Principles, Characterization, and Processing, Guo, Q., Ed. John Wiley \& Sons, Inc: Hoboken, NJ, 2016.

(62) Avrami, M. Kinetics of Phase Change. I General Theory. The Journal of Chemical Physics 1939, 7, 1103-1112.

(63) Avrami, M. Kinetics of Phase Change. Ii Transformation-Time Relations for Random Distribution of Nuclei. The Journal of Chemical Physics 1940, 8, 212-224. 
(64) Avrami, M. Granulation, Phase Change, and Microstructure Kinetics of Phase Change. Iii. The Journal of Chemical Physics 1941, 9, 177-184.

(65) Kolmogorov, A. N. Zur Statistik Der KristallisationsvorgäNge in Metallen. Izv. Akad. Nauk SSSR Ser. Mat. 1937, 1, 355-359.

(66) Johnson, W. A.; Mehl, R. F. Reaction Kinetics in Processes of Nucleation and Growth. Transactions of American Institute of Mining and Metallurgical Engineers 1939, 135, 416458.

(67) Schultz, J. M., Polymer Crystallization. Oxford: 2001.

(68) Palacios, J. K.; Tercjak, A.; Liu, G.; Wang, D.; Zhao, J.; Hadjichristidis, N.; Müller, A. J. The trilayered morphology of an $\mathrm{ABC}$ triple crystalline triblock terpolymer. Macromolecules 2017, accepted.

(69) Arnal, M. L.; Boissé, S.; Müller, A. J.; Meyer, F.; Raquez, J. M.; Dubois, P.; Prud'Homme, R. E. Interplay between Poly(Ethylene Oxide) and Poly(L-Lactide) Blocks During Diblock Copolymer Crystallization. CrystEngComm 2016, 18, 3635-3649. 


\section{Supporting Information}

\section{How the complex interplay between different blocks \\ determines the isothermal crystallization kinetics of triple crystalline PEO-b-PCL-b-PLLA triblock terpolymers}

Jordana K. Palacios ${ }^{\mathrm{a}}$, Junpeng Zhao ${ }^{\mathrm{b}}$, Nikos Hadjichristidis ${ }^{\mathrm{c}}$, Alejandro J. Müller ${ }^{\mathrm{a}, \mathrm{d} *}$

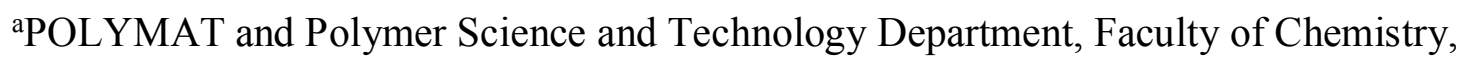
University of the Basque Country UPV/EHU, Paseo Manuel de Lardizabal 3, 20018 Donostia-San Sebastián, Spain..

${ }^{b}$ Faculty of Materials Science and Engineering, South China University of Technology, Guangzhou 510640, People's Republic of China

${ }^{\mathrm{c}}$ King Abdullah University of Science and Technology (KAUST), Physical Sciences and Engineering Division, KAUST Catalysis Center, Thuwal, Saudi Arabia.

dIKERBASQUE, Basque Foundation for Science, Bilbao, Spain.

* Corresponding authors

E-mail: alejandrojesus.muller@ehu.es 

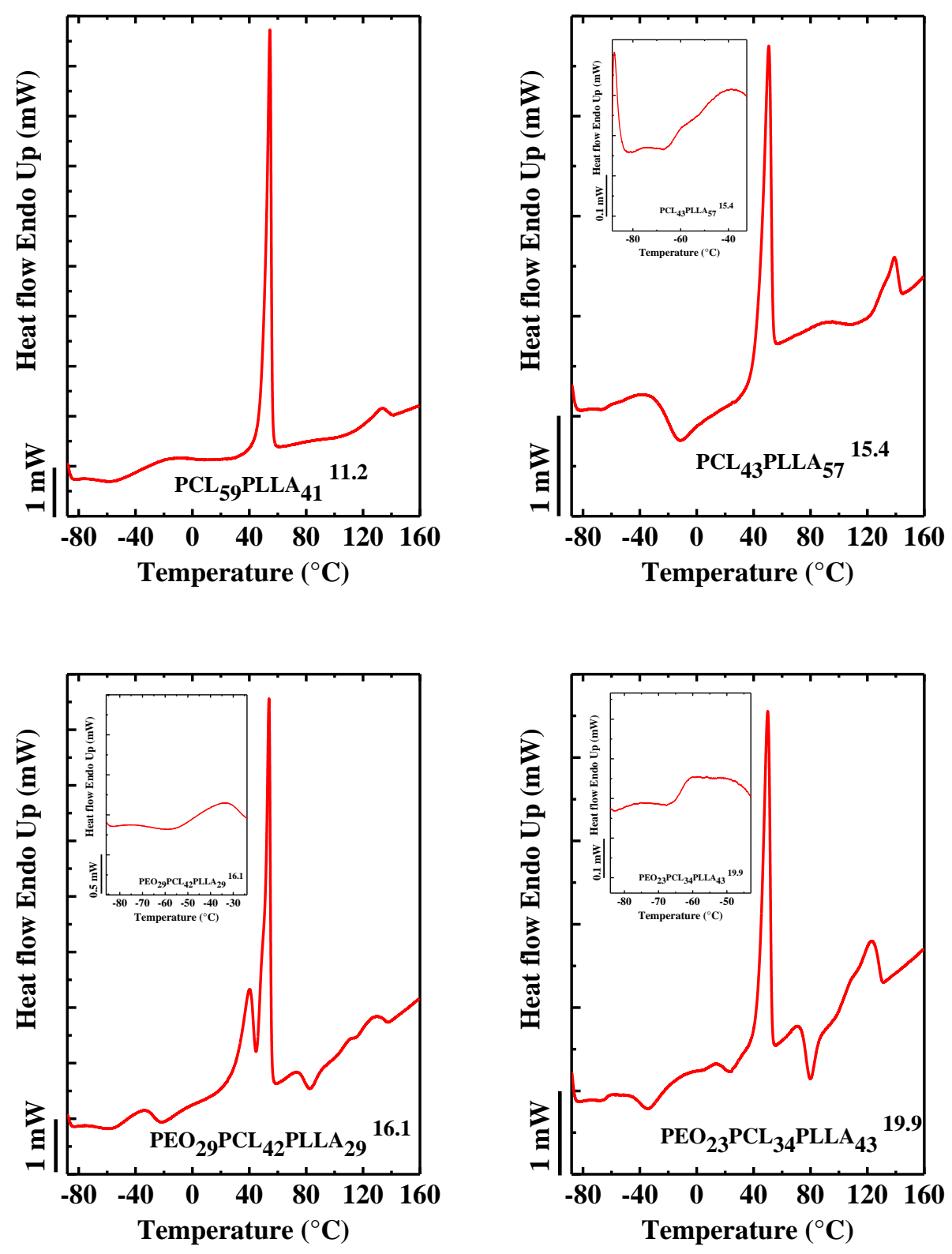

Figure S1. DSC subsequent heating scans at $20^{\circ} \mathrm{C} \mathrm{min}^{-1}$ after quenching the samples from the melt. 


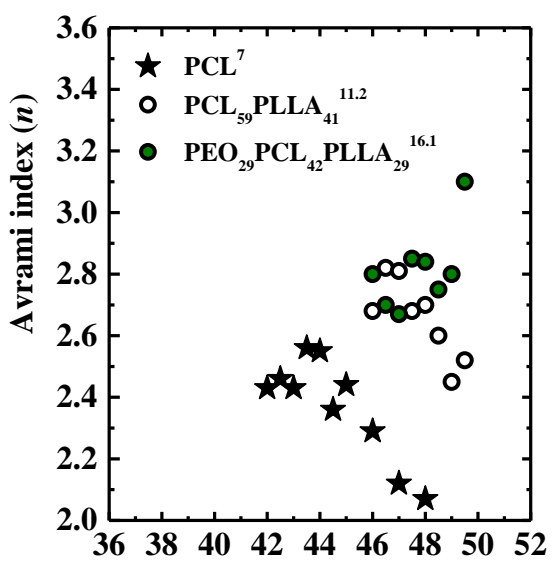

Crystallization temperature $\left({ }^{\circ} \mathrm{C}\right)$

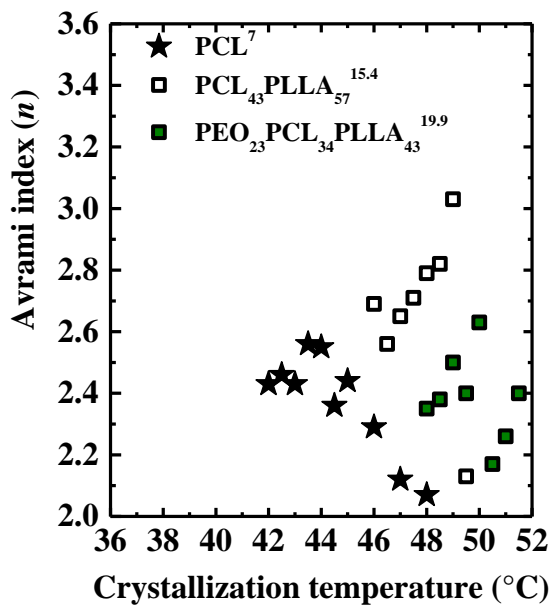

Figure S2. Avrami index values of the PCL homopolymer and PCL block within the diblock copolymers and terpolymers indicated, after first isothermally crystallizing the PLLA block until saturation.
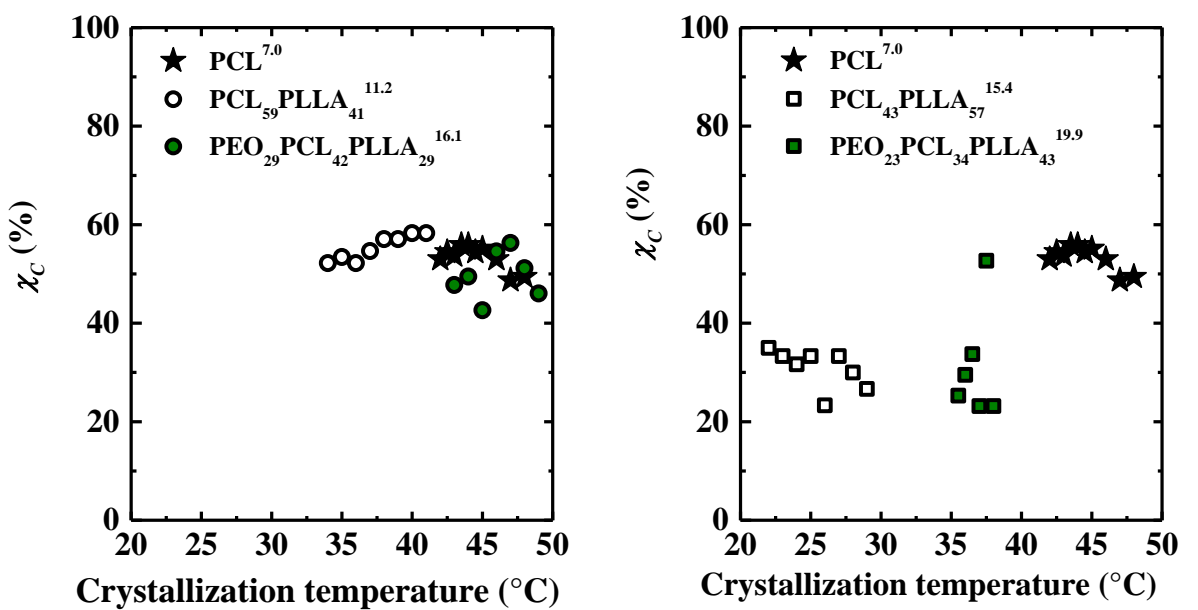

Figure S3. Crystallization degree $\left(\chi_{c}\right)$ for the PCL block within the diblock copolymers and terpolymers indicated, crystallized in one step (protocol 2). 


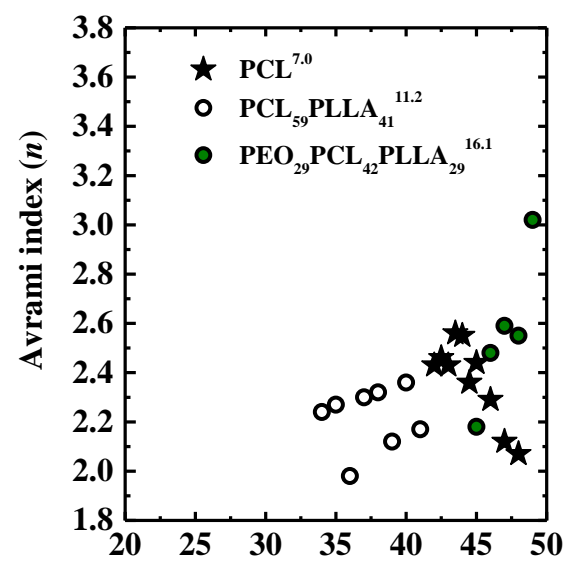

Crystallization temperature $\left({ }^{\circ} \mathrm{C}\right)$

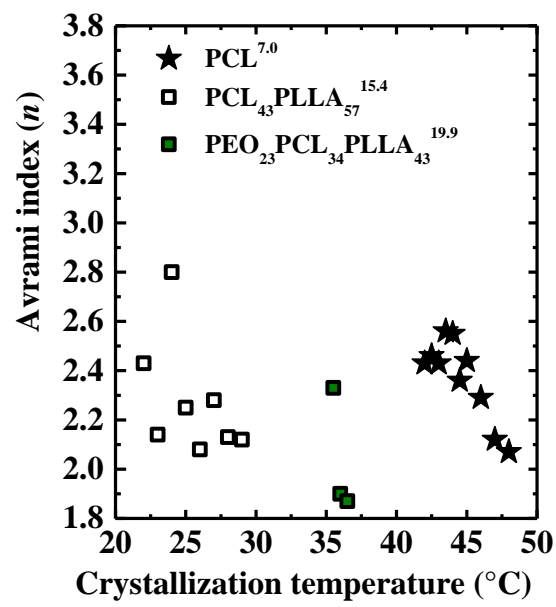

Figure S4. Avrami index values of the PCL homopolymer and PCL block within the diblock copolymers and terpolymers indicated, crystallized in one step (protocol 2).

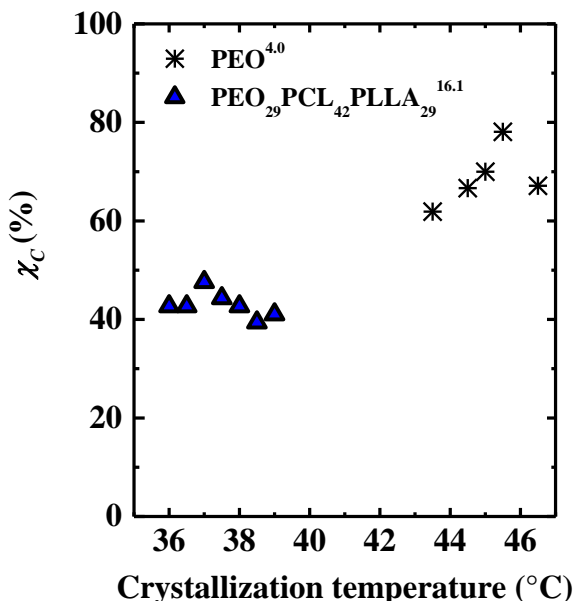

Figure S5. Crystallization degree $\left(\chi_{c}\right)$ of the PEO hompolymer and the PEO block within the $\mathrm{PEO}_{29} \mathrm{PCL}_{42} \mathrm{PLLA}_{29}{ }^{16.1}$ triblock terpolymer. 


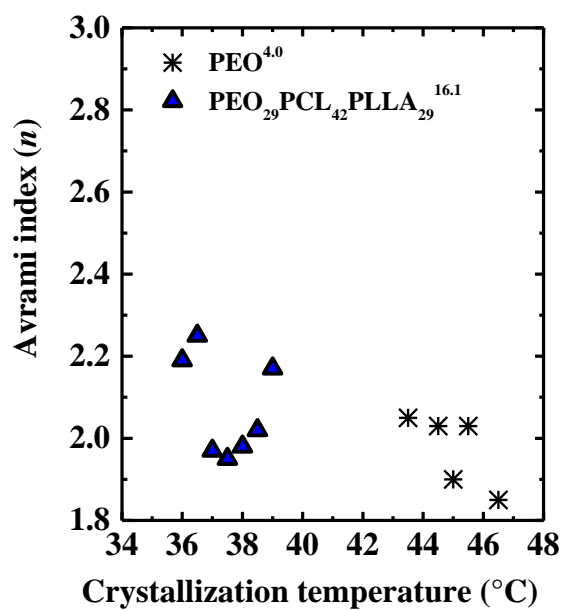

Figure S6. Avrami index as function of crystallization temperature for the PEO homopolymer and the PEO block within the $\mathrm{PEO}_{29} \mathrm{PCL}_{42} \mathrm{PLLA}_{29}{ }^{16.1}$ triblock terpolymer. 
"for Table of Contents use only"

\section{How the complex interplay between different blocks determines the isothermal crystallization kinetics of triple crystalline PEO-b-PCL-b-PLLA triblock terpolymers}

Jordana K. Palacios ${ }^{a}$, Junpeng Zhao ${ }^{b, c}$, Nikos Hadjichristidis ${ }^{c *}$, Alejandro J. Müller $^{a, d *}$

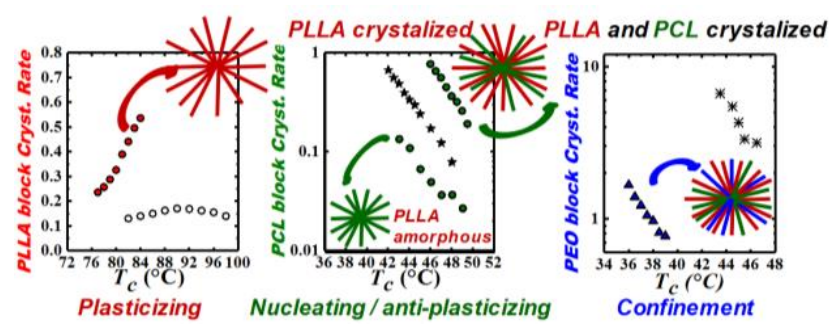

